\title{
Tin versus Lead Redox Chemistry Modulates Charge Trapping and Self-Doping in Tin/Lead Iodide Perovskites
}

Daniele Meggiolaro, ${ }^{a}{ }^{*}$ Damiano Ricciarelli, ${ }^{\mathrm{a}, \mathrm{b}}$ Ahmed A. Alasmari, ${ }^{\mathrm{c}, \mathrm{d}}$ Fatmah A. S. Alasmary, ${ }^{\mathrm{e}}$ Filippo De Angelis ${ }^{\mathrm{a}, \mathrm{b}, \mathrm{e}, *}$
a. Computational Laboratory for Hybrid/Organic Photovoltaics (CLHYO)Istituto CNR di Scienze e Tecnologie Chimiche "Giulio Natta"(CNR-SCITEC) Via Elce di Sotto 8, 06123Perugia, Italy.
b. Department of Chemistry, Biology and Biotechnology, University of Perugia,Via Elce di Sotto8, 06123 Perugia, Italy.
c. The First Industrial Institute, TVTC, Riyadh11451, Saudi Arabia. dPhysics and Astronomy Department, College of Science, King Saud University, Riyadh11451, Saudi Arabia.
e. ChemistryDepartment, College of Science, King Saud University, Riyadh11451, Saudi Arabia.

\section{E-mail: daniele.meggiolaro@cnr.it, filippo@thch.unipg.it}
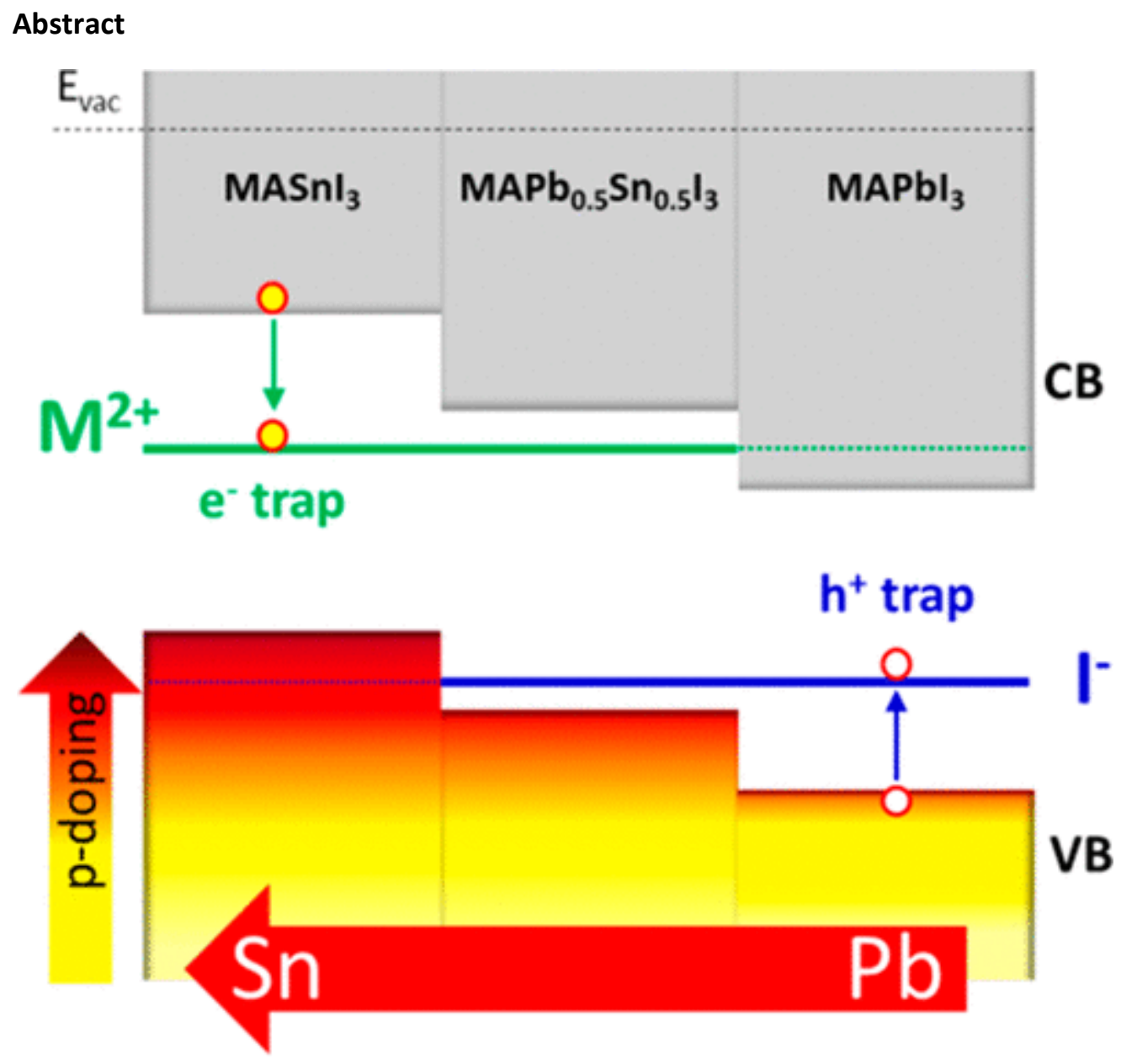

Tin halide perovskites make up the only lead-free material class endowed with optoelectronic properties comparable to those of lead iodide perovskites. Despite significant progress, the device efficiency and stability of tin halide perovskites are still limited by two potentially related phenomena, i.e., self-p-doping and tin oxidation. Both processes are likely related to defects; thus, understanding tin halide defect chemistry is a key step toward exploitation of this class of materials. We investigate the MASnl 3 perovskite defect chemistry, as a prototype of the entire materials class, using state-of-the-art density functional theory simulations. We show that the inherently low ionization potential of $\mathrm{MASnl}_{3}$ is solely responsible of the high stability of tin vacancy and interstitial iodine defects, which are in turn at the origin of the material p-doping. Tin vacancies create a locally iodine-rich environment that could promote $\mathrm{Sn}(\mathrm{II}) \rightarrow \mathrm{Sn}(\mathrm{IV})$ 
oxidation. The higher band edge energies of $\mathrm{MASnl}_{3}$ compared to those of $\mathrm{MAPbl}_{3}$ lead to the emergence of deep electron traps associated with undercoordinated tin defects (e.g., interstitial tin) and the suppression of deep transitions associated with undercoordinated iodine defects that are typical of $\mathrm{MAPbl}_{3}$. Thus, while lead iodide perovskites are dominated by iodine chemistry, tin chemistry dominates tin iodide perovskite defect chemistry. Mixed tin/lead perovskites exhibit an intermediate behavior and are predicted to be potentially free of deep traps. Compositional alloying with different metals is finally explored as a strategy for mitigating defect formation and self-p-doping in tin iodide perovskites.

Lead halide perovskites (LHPs) are outstanding materials for photovoltaics due to their high absorption coefficients, long lifetimes of photogenerated carriers, and defect tolerance.(1-6) Despite the fairly high defect density $\left(\sim 10^{15} \mathrm{~cm}^{-3}\right)$, LHP thin films have recently reached $25.2 \%$ efficiency in single-junction solar cells,(7) which rivals the efficiencies of high-purity, high-quality inorganic semiconductors. The origin of the high defect tolerance of this class of materials has been widely investigated. Density functional theory (DFT) calculations showed that many native defects in LHPs have a shallow character with thermodynamic ionization levels close to or inside the band edges with a negligible impact on the optoelectronic properties of these materials. $(8,9)$ Subsequent studies highlighted the existence of deep traps in the prototypical $\mathrm{MAPbl}_{3}(\mathrm{MA}=$ methylammonium) perovskite that are associated with undercoordinated iodine atoms (e.g., interstitial iodine and lead vacancies) located in the bulk and at the surface and grain boundaries of polycrystalline thin films. $(6,10)$ Significantly, these defects only marginally impact the lifetime of the photogenerated charge carriers due to the existence of thermodynamic and kinetic barriers to trapping and recombination. $(6,11)$

Despite the excellent properties of LHPs, concerns about lead toxicity $(12,13)$ have led to an increasing level of interest in replacing lead with less toxic metals.(14) $\mathrm{Sn}(\mathrm{II}),(15,16) \mathrm{Ge}(\mathrm{II}),(17)$ and combinations of I/III metals like $\mathrm{Bi}$ and $\mathrm{Ag}(18-20)$ have been proposed as substitutes for lead in halide perovskites. Among these materials, however, only tin halide perovskites have shown significant photovoltaic properties, with device efficiencies reaching $\sim 10 \%$. $15,21-23)$ The use of tin in combination with lead in mixed tin/lead perovskites is so far the most effective strategy for reducing the lead content in perovskite thin films; with efficiencies of $\sim 17 \%$, these mixed perovskites can be competitive with pure LHPs.(24) A further advantage of using tin and mixed tin/lead iodide perovskites is their lower and tunable band gap ( 1.1-1.3 eV, by varying the ratio of the two metals) compared to those of typical LHPs $(\sim 1.5-1.6 \mathrm{eV})$, coupled to lower exciton binding energies.(25) These characteristics make mixed tin/lead perovskites appealing for the development of efficient all-perovskite tandem solar cells.(22)

The device efficiency of tin perovskites is strongly limited by the self-p-doping characteristic of such materials that increase the background hole density to intolerable values of $\sim 10^{20} \mathrm{~cm}^{-3}$. (16) In fact, $\mathrm{MASnl}_{3}$ was initially thought to be metallic due to the strong $p$-doping leading to unusually high conductivity.(26) Similarly, $\mathrm{CsSnl}_{3}$ was initially proposed as a solid state hole transporter in dye-sensitized solar cells by virtue of its high p-type conductivity.(27) P-Doping increases recombination rates, reducing dramatically the lifetime and diffusion length of photogenerated charge carriers by more than an order of magnitude compared to the typical values observed in LHPs.(25)

In addition to p-doping, and possibly related to it, the facile oxidation of Sn(II) to Sn(IV) limits the air stability of polycrystalline thin films, $(15,25)$ promoting the degradation of the perovskite to $\mathrm{Sn}$ (IV)-based secondary phases such as $\mathrm{MA}_{2}\left(\mathrm{FA}_{2}\right) \mathrm{Snl}_{6}$ and $\mathrm{Snl}_{4}(\mathrm{FA}=$ formamidinium).(28,29) To alleviate this issue, Shao et al. used three-dimensional (3D)/two-dimensional (2D) mixed dimensionality perovskites that stabilize the 3D $\mathrm{FASnl}_{3}$ phase by interfacing it with a more stable 2D PEA $\mathrm{FA}_{n-1} \mathrm{Sn}_{n} I_{n+1}$ phase (PEA = phenylethylammonium), possibly passivating surface defects and grain boundaries. $(30)$ Insight into the degradation mechanisms of $\mathrm{FASnl}_{3}$ and $\mathrm{FAPb}_{0.5} \mathrm{Sn}_{0.5} \mathrm{I}_{3}$ has been provided by Leijtens et al. through thermogravimetric analysis in air, X-ray diffraction, and ultraviolet-visible spectroscopy.(31) The oxidation of $\mathrm{FASnl}_{3}$ proceeds with release of $\mathrm{FAl}, \mathrm{SnO}_{2}$, and $\mathrm{Snl}_{4}$, while for mixed tin/lead perovskites, release of 
$\mathrm{I}_{2}$ was also observed in addition to that of $\mathrm{FAl}, \mathrm{SnO}_{2}$, and $\mathrm{PbI}_{2}$.(31) While $\mathrm{I}_{2}$ and $\mathrm{PbI}_{2}$ are typical degradation products of LHPs, formation of $\mathrm{PbO}_{2}$ is less likely to occur in LHPs due to the higher oxidation potential of the $\mathrm{Pb}(\mathrm{II}) / \mathrm{Pb}$ (IV) redox couple compared to that of the $\mathrm{Sn}(\mathrm{II}) / \mathrm{Sn}$ (IV) couple. As a side note, but relevant to our modeling study, the difference in lead and tin redox potentials is dictated by relativistic effects, such as spin-orbit coupling (SOC),(32) which are a key ingredient, together with hybrid functionals, of accurate simulations. $(10,33)$

Several experimental strategies have been elaborated to reduce the level of native $p$-doping of tin halide perovskites. The addition of $\mathrm{SnF}_{2}$ has been shown to effectively stabilize the metal sublattice by decreasing the background hole density and dark current. $(25,28)$ Milot et al. reported a 10 -fold increase in the charge carrier diffusion length and lifetime for $\mathrm{FASnl}_{3}$ by addition of an excess of a $10 \%$ molar amount of $\mathrm{SnF}_{2}$ with respect to $\mathrm{Snl}_{2}$ in the precursor solution, which leads to a decrease in hole density of $\sim 2$ orders of magnitude, giving a value of $\sim 10^{18} \mathrm{~cm}^{-3}$. (25) Interestingly, although carrier dynamics in pristine and $\mathrm{SnF}_{2}-$ doped $\mathrm{FASnl}_{3}$ is still dominated by charge recombination with background holes, a remnant contribution associated with trap-mediated recombination was also reported, indicating the existence of traps of different natures that are not (completely) passivated by $\mathrm{SnF}_{2}$ treatment.(25) The addition of electrondonating and tin-complexing molecules was also shown to limit the $\mathrm{Sn}$ (II) to $\mathrm{Sn}$ (IV) oxidation, improving the perovskite morphology and the associated device efficiency. Lee et al. obtained homogeneous crystal grain growth with a reasonable $4.8 \%$ efficiency by using a combination of $\mathrm{SnF}_{2}$ and pyrazine;(34) pyrazine can form a complex with $\mathrm{SnF}_{2}$ by increasing its level of dispersion within the perovskite thin film, leading to more homogeneous films and mitigating phase separation induced by excess $\mathrm{SnF}_{2}$. Hoshi et al. demonstrated that the addition of 5 -ammonium valeric acid iodide (5-AVAl) to MASnl ${ }_{3}$ significantly improves the stability of the material in air,(35) an effect further confirmed by Tai et al. by employing hydroxybenzenesulfonic acids.(36) Song et al. demonstrated that the use of hydrazine reducing vapors, along with $\mathrm{SnF}_{2}$, causes a $20 \%$ decrease in the $\mathrm{Sn}(\mathrm{IV}) / \mathrm{Sn}(\mathrm{II})$ ratio, yielding a PCE of $3.89 \%$.(37) The $\mathrm{Sn}(\mathrm{IV}) / \mathrm{Sn}$ (II) ratio was shown to decrease using hypophosphorous acid,(38) 5-AVAI,(39) and other bulky organic cations such as ethylenediammonium di-iodide and butylammonium iodide.(40) The addition of bulky cations in particular promotes the crystallization of 2D perovskites, and even for small additions in the precursors, a significant increase in the tin perovskite phase stability is reported, likely associated with the passivation of the grain surface and boundaries preventing, among other effects, the incorporation of oxygen and moisture.(41)

Due to their strong impact on the efficiency and stability of tin halide perovskites, it is essential to investigate the microscopic origin of self-p-doping and the mechanisms leading to tin oxidation to propose solutions to both issues, possibly promoting the widespread use of lead-free perovskite solar cells. In both p-doping and tin oxidation, defects are likely to play a central role, determining both the intrinsic charge carrier density and lattice instability. To quantitatively assess the role of defects in determining $p$-doping and instability in tin halide perovskites, we investigate the thermodynamic stability and trapping activity of native defects in the prototypical $\mathrm{MASnl}_{3}$ system by state-of-the-art DFT calculations based on hybrid functionals and SOC. Our analysis shows that the high stability of tin vacancies, already recognized in previous studies, $(23,42,43)$ and interstitial iodine are responsible for the severe $p$-doping exhibited by $\mathrm{MASnl}_{3}$, which we directly relate to the inherently low ionization potential of tin iodide perovskites. By comparing the electronic structure of tin and lead iodide perovskites, we show that the strong modulation of band edge energies induced by varying the tin content significantly affects the stability of metal vacancies. The higher band edge energies (lower ionization potential) of $\mathrm{MASnl}_{3}$ compared to those of $\mathrm{MAPbl}_{3}$ largely stabilize acceptor defects and lead to the emergence of deep electron traps associated with undercoordinated tin defects (e.g., interstitial tin) and the suppression of deep transitions associated with undercoordinated iodine defects that are active in LHPs. The shift of the band edge energies predicted upon moving from $\mathrm{MASnl}_{3}$ to $\mathrm{MAPbl}_{3}(44)$ places the mixed $\mathrm{MAPb}_{0.5} \mathrm{Sn}_{0.5} \mathrm{l}_{3}$ phase in an intermediate regime between the pure tin and lead phases. A high concentration of tin vacancies may also create a locally 
iodine-rich environment that could promote $\mathrm{Sn}(\mathrm{II}) \rightarrow \mathrm{Sn}$ (IV) oxidation through the formation of a vacancyordered phase similar to typical $\mathrm{Sn}(\mathrm{IV})$ phases, e.g., $\mathrm{MA}_{2} \mathrm{SnI}_{6}$.(43) While extrinsic factors, such as exposure to oxidizing agents $\left(\mathrm{O}_{2}\right.$ or $\left.\mathrm{I}_{2}\right)$, can be limited by caution in the processing conditions and encapsulation, the intrinsic instability related to the electronic structure of the material remains a critical issue that can be managed by high-quality crystal grain growth and proper surface passivation treatments. In addition, we propose a strategy for alleviating the instability of tin iodide perovskites by alloying tin with different metals.

The thermodynamics and trapping activity of native defects are investigated by calculating the associated defect formation energies (DFEs) and thermodynamic ionization levels (TILs), $\varepsilon\left(q / q^{\prime}\right)$, in the supercell approach $(33,45)$ (see Computational Details). DFEs provide information about the stability of a given defect and, thus, its thermodynamic propensity to form. TILs basically represent redox potentials associated with carrier trapping at defect sites, whereby a TIL placed (deep) in the material gap will trap a carrier; a defect with a TIL within the band edges will be essentially harmless in terms of trapping but may dope the material if its charge is not compensated by defects of the opposite charge. To provide a reliable description of defect equilibrium geometries in various charge states, $(33)$ the PBEO hybrid functional $(46,47)$ was used for structural relaxations, including D3 dispersion corrections. (48) Single-point HSE06-SOC $(\alpha=$ 0.43)(49) calculations were performed on the PBEO-D3 optimized structures to obtain accurate band and defect energetics (see Computational Details).(33) All calculations have been carried out considering the respective tetragonal phases of $\mathrm{MAPb}_{x} \mathrm{Sn}_{1-x} \mathrm{l}_{3}$ perovskites $(x=0,0.5$, and 1$)$.

The calculated thermodynamic stability field of $\mathrm{MASnl}_{3}$ is compared to that of $\mathrm{MAPbl}_{3}$ in Figure 1 , which illustrates the range of chemical potentials of the constituent elements in which the possible phases resulting from combination of these elements are stable. $\mathrm{MAPbl}_{3}$ is stable in a narrow region of chemical potentials, enclosed between the $\mathrm{Pbl}_{2}$ and MAl precursors, and it may exist in thermodynamic equilibrium with solid $I_{2}$ (Figure 1a). MASnl ${ }_{3}$ is also stable in a narrow region between $\mathrm{SnI}_{2}$ and MAI precursors, with a comparable formation enthalpy (-0.07 and $-0.14 \mathrm{eV}$ for $\mathrm{MAPbl}_{3}$ and $\mathrm{MASnl}_{3}$, respectively). Under iodinerich conditions $\left(\Delta \mu_{1}>-0.16 \mathrm{eV}\right)$, however, $\mathrm{MASnl}_{3}$ is no more stable and spontaneously transforms into $\mathrm{MA}_{2} \mathrm{Snl}_{6}$ and $\mathrm{Snl}_{4}$ phases characterized by oxidation of $\mathrm{Sn}(\mathrm{II})$ to $\mathrm{Sn}(\mathrm{IV})$.(43) The existence of thermodynamically stable $\mathrm{Sn}(\mathrm{IV})$ phases, not existing for $\mathrm{MAPbl}_{3}$, represents an inherent stability limit of $\mathrm{MASnl}_{3}$ (and in general of all tin perovskites) $(42,43)$ with respect to LHPs, which becomes more relevant in oxidative environments, e.g., in the presence of $\mathrm{I}_{2}$.

\section{Figure 1}

a)

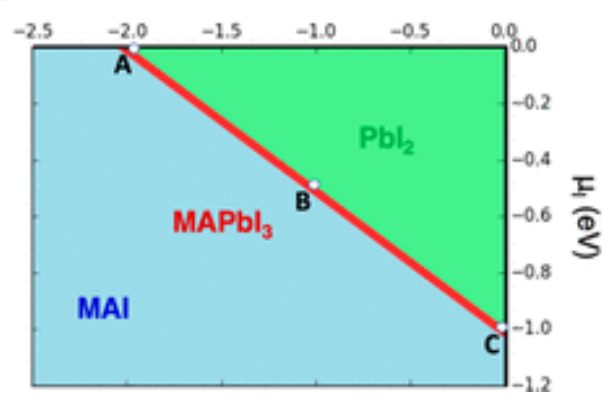

b)

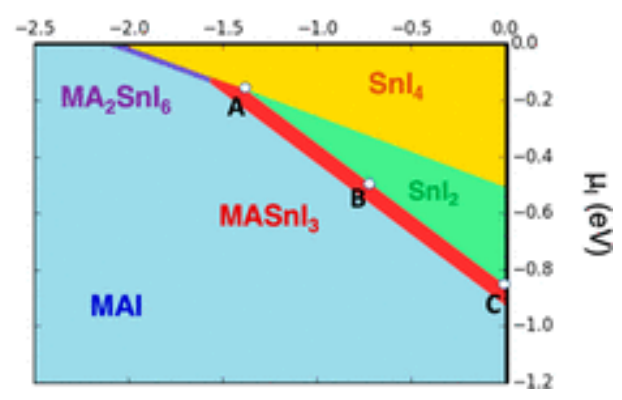

Figure 1. Phase diagrams of (a) $\mathrm{MAPbl}_{3}$ and (b) MASnl ${ }_{3}$ calculated the HSEO6-SOC ( $\alpha=0.43$ ) level of theory, including dispersion corrections. The chemical potentials of $\mathrm{Sn}, \mathrm{Pb}$, and I have been referenced to metallic $\mathrm{Sn}$, metallic $\mathrm{Pb}$, and solid $\mathrm{I}_{2}$, respectively. Three chemical potential points on the equilibrium lines between the perovskites and the relative precursors have been highlighted, indicating I-rich (point $A, \Delta \mu_{1}=$ $0.0 \mathrm{eV}$ and $\Delta \mu_{\mathrm{Pb}}=-1.99 \mathrm{eV}$ for $\mathrm{MAPb}_{3} ; \Delta \mu_{1}=-0.16 \mathrm{eV}$ and $\Delta \mu_{\mathrm{Sn}}=-1.39 \mathrm{eV}$ for MASnl ${ }_{3}$ ), I-medium (point B, $\Delta \mu_{1}=-0.50 \mathrm{eV}$ and $\Delta \mu_{\mathrm{Pb}}=-1.00 \mathrm{eV}$ for $\mathrm{MAPbl}_{3} ; \Delta \mu_{1}=-0.51 \mathrm{eV}$ and $\Delta \mu_{\mathrm{Sn}}=-0.70 \mathrm{eV}$ for $\mathrm{MASnl}_{3}$ ), and I-poor 
(point $\mathrm{C}, \Delta \mu_{\mathrm{l}}=-1.00 \mathrm{eV}$ and $\Delta \mu_{\mathrm{Pb}}=0.00 \mathrm{eV}$ for $\mathrm{MAPbl}_{3} ; \Delta \mu_{\mathrm{l}}=-0.86 \mathrm{eV}$ and $\Delta \mu_{\mathrm{sn}}=0.00 \mathrm{eV}$ for MASnl $\mathrm{M}_{3}$ ) conditions of perovskite growth.

DFEs calculated for $\mathrm{MASnl}_{3}$ in I-rich, I-medium, and I-poor growth conditions (corresponding to stability points $\mathrm{A}-\mathrm{C}$ in Figure 1b, respectively) are reported in Figure 2. A remarkable stability of acceptor defects, i.e., tin vacancies and interstitial iodine, is found under all growth conditions. Tin vacancies are stable in the 2- charge state $\left(\mathrm{V}_{\mathrm{Sn}^{2}}{ }^{2-}\right)$, with a 2-/- transition resonant with the top of the valence band (VB) (see Figure 3a). Upon removal of Sn from its crystal site, the lattice undergoes a limited rearrangement, leaving the vacancy site almost intact (see the optimized structures in Figure 4a). Similarly, interstitial iodine is stable in the 1- charge state $\left(\mathrm{I}_{\mathrm{i}}^{-}\right)$with the $-/ 0$ transition resonant with the top of the VB (Figure 3a). Interstitial iodine shows the typical bridge structure found for $\mathrm{MAPbl}_{3}$ (Figure 4b).(10)

\section{Figure 2}
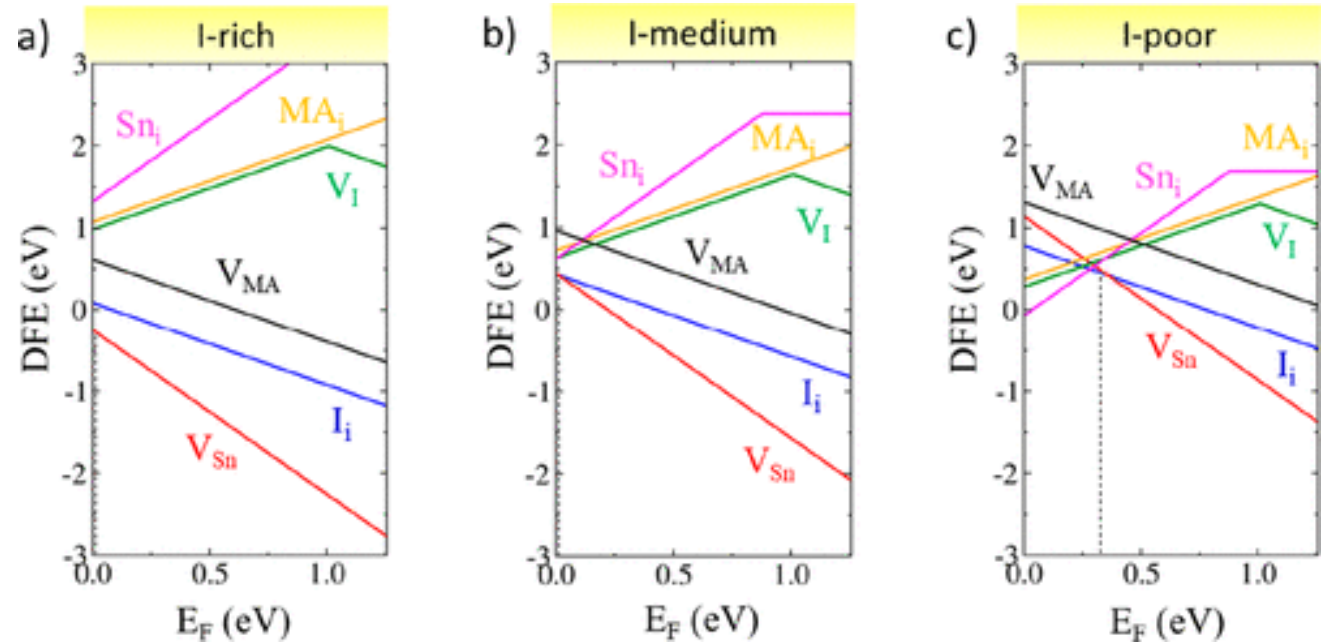

Figure 2. Defect formation energy diagrams of native defects in MASnl 3 calculated at the HSE06-SOC-D3 ( $\alpha$ $=0.43$ ) level of theory for (a) I-rich, (b) I-medium, and (c) I-poor chemical conditions, i.e., points A-C in Figure $1 b$, respectively. The vertical dashed line, overlapping with the valence band in panels $a$ and $b$, indicates the native Fermi level of the system.

\section{Figure 3}
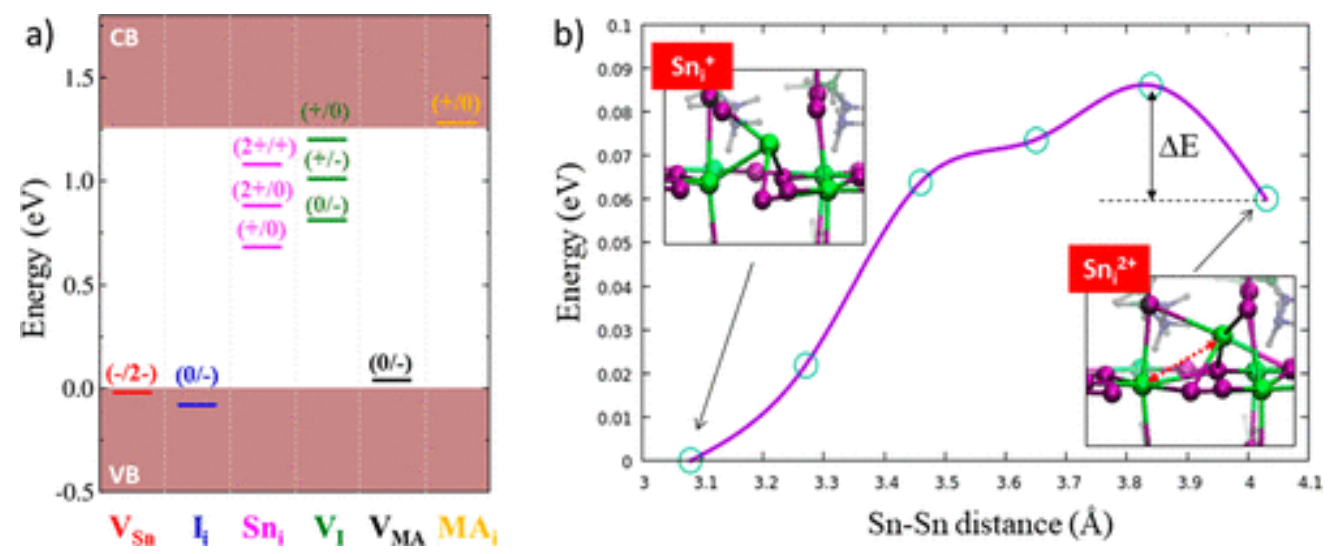

Figure 3. (a) Thermodynamic ionization levels of native defects in $\mathrm{MASnl}_{3}$ calculated at the HSEO6-SOC-D3 $\left(\alpha=0.43\right.$ ) level of theory. (b) Simulated path for electron trapping at the $\mathrm{Sn}_{\mathrm{i}}{ }^{2+}$ defect. Tin ions are unbound in the $\mathrm{Sn}_{\mathrm{i}}{ }^{2+}$ defect (right), while they are bonded to form a dimer in the $\mathrm{Sn}_{i}^{+}$equilibrium configuration (left). The calculated barrier $\Delta E$ is 0.03 and $0.10 \mathrm{eV}$ at the PBE and HSE06-SOC levels of theory, respectively. 
Figure 4

a)

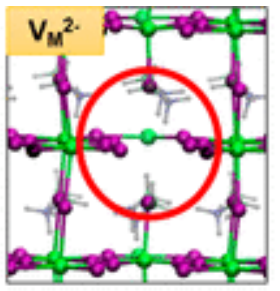

e)

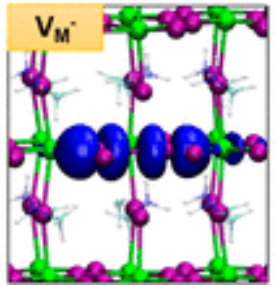

b)

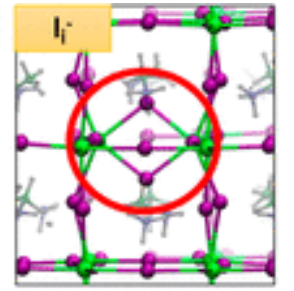

f)

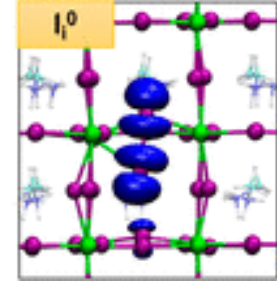

c)

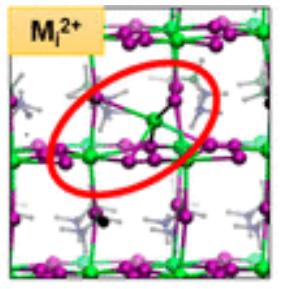

g)

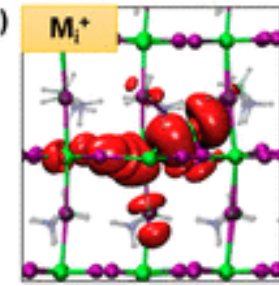

d)

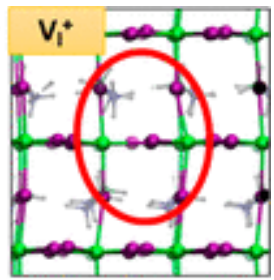

h)

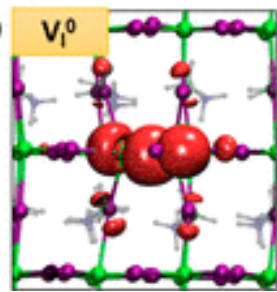

Figure 4. (a-d) Optimized structures of the main defects in $\mathrm{MASnl}_{3}$ and $\mathrm{MAPbl}_{3}$ (green for metal and purple for iodine). $\mathrm{Sn}$ and $\mathrm{Pb}$ are both denoted as $\mathrm{M}$. (e-f) Plot of the orbital associated to the trapped charge on the $\mathrm{V}_{\mathrm{M}}{ }^{-}$and $\mathrm{l}_{\mathrm{i}}{ }^{0}$ defects in $\mathrm{MAPbl}_{3}$. (g-h) Plot of the orbital associated to the trapped electron on the $\mathrm{M}_{\mathrm{i}}{ }^{+}$and $\mathrm{V}_{1}^{0}$ defects in $\mathrm{MASnl}_{3}$.

$\mathrm{MASnl}_{3} \mathrm{p}$-doping is thus promoted by the formation of both defects, following the reactions

$$
\begin{aligned}
& \mathrm{Sn}_{\mathrm{Sn}}=\mathrm{Sn}_{\text {bulk }}+\mathrm{V}_{\mathrm{Sn}^{2-}}+2 \mathrm{~h}^{+} \\
& \frac{1}{2} \mathrm{I}_{2}=\mathrm{I}_{\mathrm{i}}^{-}+1 \mathrm{~h}^{+}
\end{aligned}
$$

In reaction 1, the reduction of $\mathrm{Sn}(\mathrm{II})$ to $\mathrm{Sn}$ metal $\left(\mathrm{Sn}_{\text {bulk }}\right)$ leads to the oxidation of the lattice by release of two holes. This process formally leads to the loss of the metal from the perovskite to form a Sn-rich phase, in agreement with experiments reporting the formation of Sn-rich clusters in mixed tin/lead perovskites with the concomitant formation of subgap trap states.(22) Similarly, in reaction 2, the incorporation of iodine to form $\mathrm{I}_{\mathrm{i}}^{-}$introduces one charge hole into the VB. It is clear that these processes are in general facilitated by a high VB energy, i.e., by a low ionization potential, which stabilizes the charge holes produced by the perovskite to compensate the negative charge of the native defects.

The lack of compensating defects with a positive charge under I-rich and I-medium conditions places the native Fermi level at the top of the $\mathrm{VB}$, indicating that $\mathrm{MASnl}_{3}$ tends to grow intrinsically $\mathrm{p}$-doped, with estimated hole densities of $\sim 10^{19} \mathrm{~cm}^{-3}$ under I-medium perovskite growth conditions, in fairly good agreement with experiments.(21) Notice that our simulations refer to perfect bulk crystals, so that higher defect (and hole) densities are expected in polycrystalline thin films due to lower DFEs typically associated with surfaces and grain boundaries. $(50)$

Donor defects, such as iodine vacancies $\left(\mathrm{V}_{1}^{+}\right)$and interstitial tin $\left(\mathrm{Sn}_{i}{ }^{2+}\right)$, are less stable than acceptor defects under I-rich and I-medium conditions (Figure 2a,b), while they show stability comparable to that of tin vacancies under I-poor conditions for which $\mathrm{Sn}_{i}{ }^{2+}$ is the most stable defect (Figure 2c). Via introduction of a stable (i.e., of low DFE) positively charged defect compensating for the negative charge of $\mathrm{V}_{\mathrm{Sn}^{2-}}{ }^{2}$ and $\mathrm{I}_{\mathrm{i}}^{-}$, the native Fermi level rises at $\sim 0.3 \mathrm{eV}$ above the VB maximum. Our analysis shows that under I-poor (or Sn- 
rich) conditions self-p-doping can be limited by formation of $\mathrm{Sn}_{i}{ }^{2+} / \mathrm{V}_{\mathrm{Sn}}{ }^{2-}$ Frenkel pairs, whose formation energy in the limit of non-interacting defects is comparable to that computed for $\mathrm{MAPbl}_{3 .}(51)$

In the stable $2+$ charge state, interstitial tin $\left(\mathrm{Sn}_{\mathrm{i}}{ }^{2+}\right)$ shows a distorted octahedral configuration, with a shortest $\mathrm{Sn}-\mathrm{Sn}$ distance of $4.08 \AA$ (Figures $3 \mathrm{~b}$ and $4 \mathrm{c}$ ). Addition of electrons to form $\mathrm{Sn}_{i}{ }^{+}$and $\mathrm{Sn}_{i}{ }^{0}$ leads to a decrease in the $\mathrm{Sn}-\mathrm{Sn}$ distance to 3.08 and $2.94 \AA$, respectively, indicating the formation of a new bond between lattice and interstitial $\mathrm{Sn}$ ions (igures $3 \mathrm{~b}$ and $\underline{4 \mathrm{~g}}$ ). The corresponding $2+/+$ and $2+/ 0$ TILs are placed 1.08 and $0.88 \mathrm{eV}$ above the VB maximum, respectively, over a calculated band gap of $1.26 \mathrm{eV}$ (Figure 3a). The thermodynamically stable $\mathrm{Sn}_{i}{ }^{2+}$ defect can thus trap electrons through the $2+/+$ transition calculated at $0.18 \mathrm{eV}$ below the conduction band (CB) minimum, promoting nonradiative recombination. This is likely the defect observed by Milot et al. leading to a remnant bimolecular recombination in FASnl 3. (25)Figure 4g shows a plot of the orbital associated with the defect-trapped electron of $\mathrm{Sn}_{i}^{+}$. Because in the $2+$ charge state no such orbital is observed in the band gap, a barrier to electron trapping at this defect is likely to occur, possibly limiting its trapping activity. To estimate the electron trapping barrier at this defect, a linear transit between the $\mathrm{Sn}_{i}{ }^{2+}$ and $\mathrm{Sn}_{i}{ }^{+}$geometrical configurations in the $1+$ state of charge was carried out (see Computational Details). The calculated path confirms the existence of a small barrier to electron trapping on the $\mathrm{Sn}_{\mathrm{i}}{ }^{2+}$ defect of $0.1 \mathrm{eV}$ at the HSEO6-SOC level (see Figure $3 \mathrm{~b}$ ). This highlights the fact that electron trapping at interstitial tin may be kinetically hindered, potentially limiting the trapping activity of this defect.

lodine vacancies are stable in the positive $\left(\mathrm{V}_{1}^{+}\right)$and negative $\left(\mathrm{V}_{1}^{-}\right)$charge states along the Fermi level. In the positively charged state, the two undercoordinated tin atoms are at distances of $\sim 6 \AA$, with minimal geometrical rearrangement compared to the pristine structure (Figure $4 \mathrm{~d}$ ). Like the case for interstitial tin, addition of electrons to $\mathrm{V}_{1}^{+}$leads to the formation of a $\mathrm{Sn}-\mathrm{Sn}$ bonded dimer, with intermetal distances of 3.48 and $3.24 \AA$ for the neutral and negative states, respectively (Figure $4 \mathrm{~h}$ ). The density plot of the trapped electron on the neutral $V_{1}{ }^{0}$, reported in Figure $4 h$, highlights the charge localization on the defect center. The calculated +/0 and +/- TILs are found 1.20 and $1.01 \mathrm{eV}$ above the VB maximum, respectively; thus, electron trapping on the $\mathrm{V}_{1}{ }^{+}$centers is only slightly favored, with a TIL lying $0.06 \mathrm{eV}$ below the CB minimum. Defects associated with the cation sublattice such as $\mathrm{VMA}^{-}$and $\mathrm{MA}_{i}{ }^{+}$, on the other hand, do not introduce deep levels in the band gap; thus, they are not active in trapping processes.

Our quantitative analysis of $\mathrm{MASnl}_{3}$ defect chemistry highlights two main factors possibly limiting the material optoelectronic quality: (i) the self-p-doping caused by the high stability of tin vacancy and interstitial iodine defects, which increases the level of monomolecular radiative recombination, and (ii) the existence of nonradiative recombination centers associated with undercoordinated tin centers in the form of tin interstitials and iodine vacancies.

The defect chemistry of tin perovskites appears thus to be profoundly different from that of $\mathrm{MAPbl}_{3}$ and analogous LHPs. Self-p-doping is not observed in $\mathrm{MAPbl}_{3}$, and iodine vacancies and lead interstitials, which are possible electron traps, are not active in LHPs because their TILs are resonant with the $\mathrm{CB}(6,33)$ (Figure 5d). It is interesting to further compare the defect properties of $\mathrm{MASnl}_{3}$ and $\mathrm{MAPbl}_{3}$ to those of the mixed $\mathrm{MAPb}_{0.5} \mathrm{Sn}_{0.5} \mathrm{l}_{3}$ perovskite (Figure 5). Besides the different metal, the stability and trapping activity of defects in their different states of charge are largely influenced by the electronic structure of the host, particularly by the absolute energy positions of the VBM and CBM. 


\section{Figure 5}
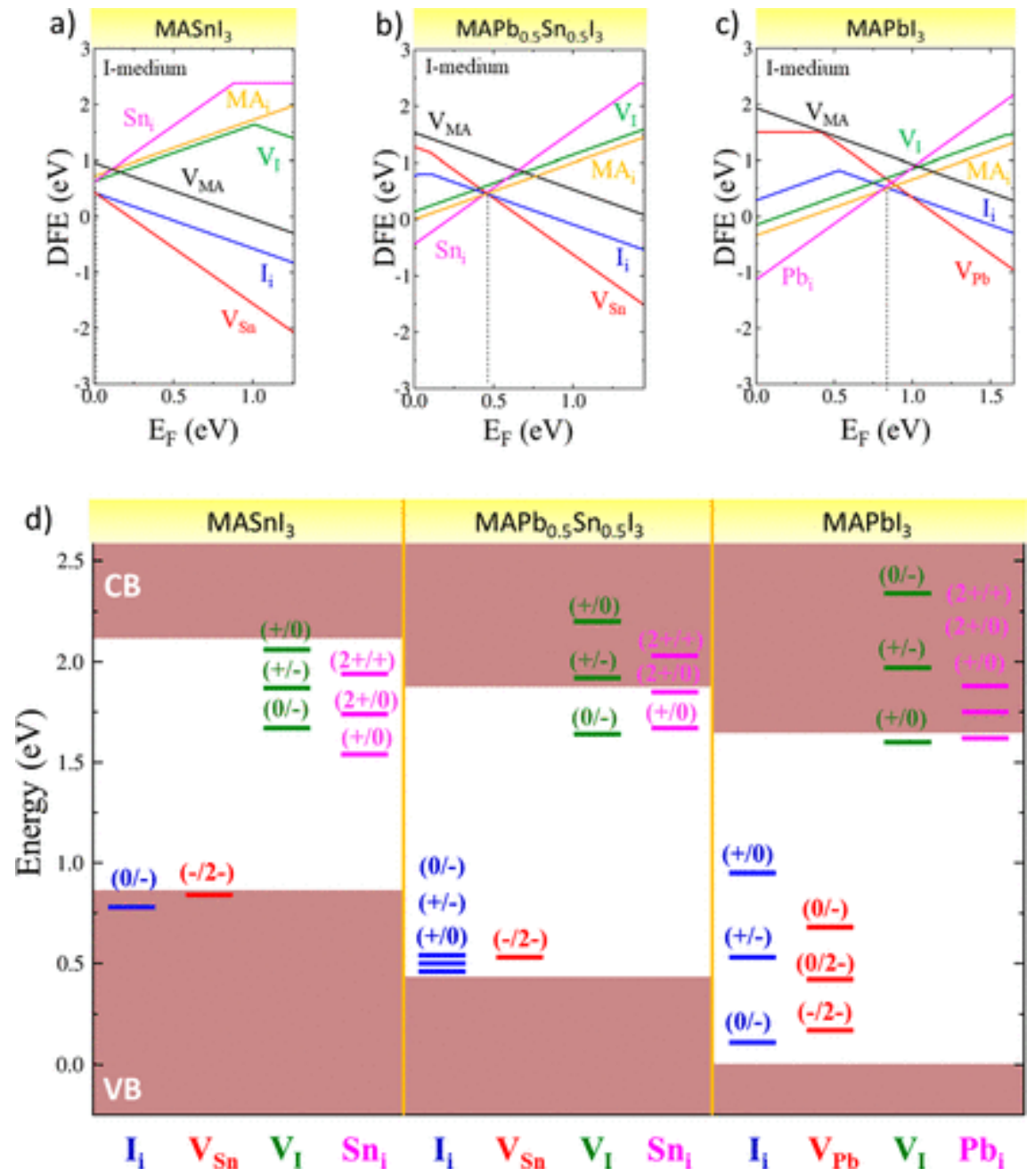

Figure 5. Defect formation energies computed at the HSE06-SOC-D3 $(\alpha=0.43)$ level of theory for I-medium conditions of (a) $\mathrm{MASnl}_{3}$, (b) $\mathrm{MAPb}_{0.5} \mathrm{Sn}_{0.5} \mathrm{I}_{3}$, and (c) $\mathrm{MAPbl}_{3}$ perovskites. (d) Thermodynamic ionization levels (TILs) of $\mathrm{MASnl}_{3}, \mathrm{MAPb}_{0.5} \mathrm{Sn}_{0.5} \mathrm{l}_{3}$, and $\mathrm{MAPbl}_{3}$ at the same level of theory. The zero of the energy scale in panel $d$ is placed at the $\mathrm{VB}$ of $\mathrm{MAPbl}_{3}$.

The VB energy ( $\varepsilon_{V B}$ in eq 3 in Computational Details) associated with the oxidation potential of the pristine material (ionization potential) defines the electronic energy associated with the exchange of charges between the defect and the Fermi level of the bulk (see Computational Details). Because an almost monotonic increase in the $\mathrm{VB}$ energy is predicted moving from $\mathrm{MAPbl}_{3}$ to $\mathrm{MAPb}_{0.5} \mathrm{Sn}_{0.5} \mathrm{I}_{3}$ and $\mathrm{MASnl}_{3}$ perovskites, we anticipate a significantly different DFE for the three perovskites, with the mixed tin/lead material showing somehow intermediate properties.

The electronic structures of tin and lead perovskites share many similarities. In both cases, the VB is composed by iodine $p$ orbitals and metal $s$ orbitals, while the $C B$ is mainly derived from metal $p$ orbitals (Figure S1). In the case of tin, the contribution of tin S orbitals to the VB is slightly larger than in the lead perovskite.(52) The different metal strongly affects the absolute position of the band edges in the two perovskites and thus the defect properties in terms of both DFEs and TILs. The calculated DFEs under Imedium growth conditions and the aligned band edges with the calculated TILs of the main defects for $\mathrm{MASnl}_{3}, \mathrm{MAPb}_{0.5} \mathrm{Sn}_{0.5} \mathrm{I}_{3}$, and $\mathrm{MAPbl}_{3}$ are compared in Figure 5. As one may notice, the absolute VBM and $\mathrm{CBM}$ energies of $\mathrm{MASnl}_{3}$ are higher, i.e., closer to the vacuum level, by 0.86 and $0.48 \mathrm{eV}$, respectively, than in $\mathrm{MAPbl}_{3}$. The mixed $\mathrm{MAPb}_{0.5} \mathrm{Sn}_{0.5} \mathrm{I}_{3}$ perovskite shows intermediate band energies between those of the full lead and full tin perovskites, with an almost linear change in the electronic structure moving between 
the two limiting compositions. The calculated band offsets are slightly higher than those measured by UPS experiments,(53) likely due to the pinning of the VBM energies caused by the different chemical compositions of the surfaces in polycrystalline thin films probed by photoemission spectroscopy.(54) It is apparent at first glance that the higher energy of $\mathrm{VBM}$ in $\mathrm{MASnl}_{3}$ with respect to that of $\mathrm{MAPbl}_{3}$ is indicative of a stronger propensity of the tin perovskite to oxidize compared to those of $\mathrm{MAPbl}_{3}$ and $\mathrm{MAPb}_{0.5} \mathrm{Sn}_{0.5} \mathrm{I}_{3}$.

Upon examination of the DFEs in panels $b$ and $c$ of Figure 5 , the most stable defects in $\mathrm{MAPbl}_{3}$ and $\mathrm{MAPb}_{0.5} \mathrm{Sn}_{0.5} \mathrm{l}_{3}$ are both donors and acceptors in the form of MA and iodine interstitials. Other defects such as $\mathrm{V}_{\mathrm{Pb}}\left(\mathrm{V}_{\mathrm{Sn}}\right)$ and $\mathrm{Pb}_{\mathrm{i}}\left(\mathrm{Sn}_{\mathrm{i}}\right)$ are also stable at the native Fermi level. Overall, a progressive stabilization (destabilization) of acceptor (donor) defects is reported moving from $\mathrm{MAPbl}_{3}$ to $\mathrm{MASnl}_{3}$ caused by the VBM upshift. The calculated DFEs show a monotonic shift moving from $\mathrm{MAPbl}_{3}$ to $\mathrm{MAPb}_{0.5} \mathrm{Sn}_{0.5} \mathrm{I}_{3}$ to $\mathrm{MASnl}_{3}$, following the shift of the electronic band edges of the pristine phases. As an example, DFEs calculated in $\mathrm{MASnl}_{3}$ at the VBM show values comparable to those calculated at an $E_{\mathrm{F}}$ of $0.86 \mathrm{eV}$ in $\mathrm{MAPbl}_{3}$, i.e., at the Fermi level corresponding to the position of the aligned VBM of $\mathrm{MASnl}_{3}$. The MAPb $0.5 \mathrm{Sn}_{0.5} \mathrm{l}_{3}$ phase shows intermediate defect properties between those of full tin and lead perovskites. This points out that contributions due to the different nature of the metal-halide bond to defect formation are rather similar and the differences in DFEs mainly stem from the different electronic structure of the pristine phases. Interestingly, native defects pin the Fermi levels $\sim 0.8, \sim 0.4$, and $\sim 0 \mathrm{eV}$ above the $\mathrm{VBM}$ in $\mathrm{MAPbl}_{3}$, $\mathrm{MAPb}_{0.5} \mathrm{Sn}_{0.5} \mathrm{l}_{3}$, and $\mathrm{MASnl}_{3}$, respectively. Self-p-doping in $\mathrm{MASnl}_{3}$ (not observed in $\mathrm{MAPb}_{3}$ ) is thus intimately related to the electronic band edge energies, whereby an increasingly intrinsic behavior can be expected upon alloying MASnl 3 with larger amounts of lead.

An interesting consequence of this behavior is that in the $p$-doped region of $\mathrm{MAPbl}_{3} \mathrm{I}_{\mathrm{i}}$ and $\mathrm{V}_{\mathrm{Pb}}$ are stable in their oxidized forms, i.e., $\mathrm{l}_{\mathrm{i}}^{+}$and $\mathrm{V}_{\mathrm{Pb}}{ }^{0}$, leading to the formation of iodine trimers whereby iodine is oxidized to a form similar to $\mathrm{I}_{3}^{-}(6,33)$ (Figure S2). Interstitial iodine and lead vacancies introduce thermodynamic ionization levels deep in the band gap and are active recombination centers in $\mathrm{MAPbl}_{3}$ (Figure $5 \mathrm{~d}$ ). $(6,33)$ The trapping of electrons and/or holes on these centers leads to the formation of intermediate radical states, i.e., $\mathrm{I}_{i}{ }^{0}$ and $\mathrm{V}_{\mathrm{Pb}}{ }^{-}$, where two iodine atoms are bonded to form dimers at distances of 3.20-3.30 $\AA$ (see panels $e$ and $f$ of Figure 4 for plots of the localized orbitals of these defects).(6,33) Similar defect structures also exist in $\mathrm{MASnl}_{3}$ but are less stable than the $\mathrm{I}_{\mathrm{i}}{ }^{-}$and $\mathrm{V}_{\mathrm{Pb}}{ }^{2-}$ defects with one and two holes delocalized in the VB, respectively; thus, the associated TILs occur deep in the VB of $\mathrm{MASnl}_{3}$. The higher VB energy of MASnl ${ }_{3}$ thus prevents their formation, by favoring only the reduced forms of these defects, i.e., $\mathrm{I}_{\mathrm{i}}{ }^{-}$and $\mathrm{V}_{\mathrm{Sn}^{2}}{ }^{2-}$, in the Fermi level range spanned by the band gap.

On the other hand, donor defects such as $\mathrm{V}_{\mathrm{I}}$ and $\mathrm{Pb}_{\mathrm{i}}$ are stable only in the respective oxidized forms in $\mathrm{MAPbl}_{3}$, i.e., $\mathrm{Pb}_{i}{ }^{2+}$ and $\mathrm{V}_{1}^{+}$. In MASnl$l_{3}$, a progressive destabilization of these defects is reported at the VBM. The higher energy of the VBM and CBM in $\mathrm{MASnl}_{3}$ compared to those of $\mathrm{MAPbl}_{3}$ stabilizes the reduced forms of these defects, i.e., $V_{i}{ }^{-}$and $\mathrm{Sn}_{\mathrm{i}}{ }^{0}$, in the $\mathrm{n}$ region of the DFE diagram, thus favoring the formation of bonded $\mathrm{Sn}$ dimers. Notably, similar structures for $\mathrm{V}_{1}{ }^{-}$and $\mathrm{Pb}_{\mathrm{i}}{ }^{0}$ are obtained in $\mathrm{MAPbl}_{3}$ at the PBE and PBEO levels of theory, (55) but the inclusion of $\mathrm{SOC}$ strongly destabilizes the respective $\mathrm{Pb}$ dimer-bonded forms, making them unstable.(33) As a result, the associated TILs that have a shallow character in $\mathrm{MAPbl}_{3}$ become deep in the band gap of $\mathrm{MASnl}_{3}$. The energy upshift of both the VBM and the CBM in MASnl ${ }_{3}$ with respect to $\mathrm{MAPbl}_{3}$ leads to the suppression of deep hole traps associated with $\mathrm{I}_{\mathrm{i}}$ and $\mathrm{V}_{\mathrm{Pb}}$ and to the emergence of deep electron traps associated with undercoordinated tin centers, i.e., $V_{1}$ and $\mathbf{S n}_{\text {i }}$. Nonradiative recombination processes in $\mathrm{MAPbl}_{3}$ and $\mathrm{MASnl}_{3}$ are thus ruled by iodine and tin chemistry, respectively. Interestingly, the band edge energies in the mixed $\mathrm{MAPb}_{0.5} \mathrm{Sn}_{0.5} \mathrm{I}_{3}$ phase, intermediate between those of $\mathrm{MAPbl}_{3}$ and $\mathrm{MASnl}_{3}$, lead to the concomitant suppression of both deep hole and electron traps associated with undercoordinated iodine and tin centers, respectively. Hence, $\mathrm{Pb}$ alloying is useful both in limiting selfp-doping and in reducing the density of recombination centers compared to that of $\mathrm{MASnl}_{3}$. 
Our analysis highlights the fact that $\mathrm{MAPb}_{3}, \mathrm{MAPb}_{0.5} \mathrm{Sn}_{0.5} \mathrm{l}_{3}$, and $\mathrm{MASnl}_{3}$ share a similar defect chemistry, but the different band edge energies modulate the relative stability of defects with transitions in the perovskite band gap. The inherent low ionization energy, promoting the formation of tin vacancies and $p$ doping, thus represents the critical factor limiting the efficiencies of tin perovskites in solar cells with respect to the mixed and full lead perovskites.

The beneficial role of Sn-rich conditions of growth in the efficiencies of tin perovskites, such as the addition of $\mathrm{SnF}_{2},(21,25,28,37,56-59)$ can be interpreted as a strategy that aims to increase the chemical potential of tin, hindering the formation of tin vacancies. (25) As reported in Figure 2c, Sn-rich (I-poor) conditions increase the DFE of $\mathrm{V}_{\mathrm{Sn}^{2-}}$ by $\sim 0.7 \mathrm{eV}$ in $\mathrm{MASnl}_{3}$ compared to stoichiometric growth conditions (I-medium). Under such conditions, the heavy p-doping of $\mathrm{MASnl}_{3}$ is mitigated by the upshifting of the native Fermi level at $\sim 0.3 \mathrm{eV}$ above the VBM due to the formation of tin Frenkel couples in the lattice. According to our predictions, a potential decrease in the hole density from $10^{19}$ to $10^{15} \mathrm{~cm}^{-3}$ may be achieved by using strong $\mathrm{Sn}$-rich conditions. The use of Sn-rich environments, on the other hand, may favor the formation of $\mathrm{Sn}_{\mathrm{i}}$ and $V_{1}$ electron traps, potentially enhancing trap-assisted recombination in the tin perovskite. The presence of a thermodynamic barrier to electron trapping on these defects, however, may attenuate their detrimental impact on the solar cell efficiency.

VB energy tuning through a change in the chemical composition may be the guideline for improving the intrinsic performance of tin-based perovskites. As discussed above, metal alloying with $\mathrm{Pb}$ is a satisfactory strategy for increasing the ionization potential of $\mathrm{MASnl}_{3}$ yielding more tolerable $\mathrm{p}$-doping and better stability, without dramatic effects on the perovskite band gap. Notably, according to our calculations, the mixing of $\mathrm{MASnl}_{3}$ and $\mathrm{MAPbl}_{3}$ to give $\mathrm{MAPb}_{0.5} \mathrm{Sn}_{0.5} \mathrm{l}_{3}$ is thermodynamically slightly disfavored by $0.04 \mathrm{eV}$, highlighting a tendency of this phase to disproportionate. This limitation can be alleviated by using tailored doping strategies, as discussed by Bowman et al., which showed that incorporation of $\mathrm{Zn}$ in mixed tin/lead perovskites induces a more controllable p-doping and an enhancement of the lattice stability.(60) Alternative strategies may rely on alkaline and transition metal doping, which have been extensively investigated in LHPs.(61-63) It has been reported that $\mathrm{Sr}$ doping of $\mathrm{FASnl}_{3}$ improves the material thermal stability and morphology, also leading to increased solar cell performances.(61-63) Like metal doping, the alloying with more electronegative halides, such as bromide, may be beneficial to increase the efficiency of tin perovskites, as shown for $\mathrm{MASnl}_{3}$ and $\mathrm{FASnl}_{3} \cdot(64,65)$

To investigate the potential effects of tin alloying with metals different from lead, we modeled $\mathrm{MASnl}_{3}$ doped with various concentrations of $\mathrm{Cd}, \mathrm{Sr}, \mathrm{Zn}$, and $\mathrm{Ca}$ as substitutions for $\mathrm{Sn}$. We monitored the shift of the band edge energies with respect to the pristine $\mathrm{MASnl}_{3}$ for doping concentrations from $\sim 3 \%$ to $25 \%$ (see the results in Table S1 and Figure S3). To provide a more accurate estimate of the VB shift, we refined calculations for the lowest dopant density of $\sim 3 \%$ at the HSE06-SOC-D3 level of theory in Table 1 .

Table 1. Energy Shifts of the VB, CB, and Band Gaps of $\mathrm{MASnl}_{3}$ Doped with $\sim 3 \% \mathrm{Cd}, \mathrm{Sr}, \mathrm{Zn}$, and Ca as Substitutions for Tin; Estimated Variations of the Energy of Formation of the Tin Vacancy ( $\mathrm{V}_{\mathrm{sn}}{ }^{2-} \mathrm{DFE}$ ) Based on the Shifts of the VB; and Calculated Exchange Energies of the Dopants as Substitutions for Tina

\begin{tabular}{llllll}
\hline metal & VB shift & CB shift & band gap shift & $\mathbf{V}_{\mathrm{sn}^{2}}{ }^{-}$DFE shift & exchange energy \\
$\mathrm{Cd}$ & -0.07 & +0.08 & +0.15 & +0.14 & -0.01 \\
\hline $\mathrm{Sr}$ & -0.06 & +0.04 & +0.10 & +0.12 & 0.03 \\
\hline $\mathrm{Zn}$ & -0.05 & +0.10 & +0.15 & +0.10 & 0.01 \\
\hline $\mathrm{Ca}$ & -0.06 & +0.05 & +0.11 & +0.12 & -0.01 \\
\hline
\end{tabular}


${ }^{a}$ Energy shifts of the $\mathrm{VB}$ and $\mathrm{CB}$ relative to the band edges of the pristine $\mathrm{MASnl}_{3}$ have been calculated after aligning the inner $4 \mathrm{~s}$ levels of tin in the perfect and doped supercells. All values (electronvolts) are calculated at the HSEO6-SOC-D3 level of theory.

As one may notice, the series of considered alloying metals confirm a decrease (increase) in the VB (CB) energy compared to that of pristine $\mathrm{MASnl}_{3}$ and an overall band gap increase. Results in Table 1 are consistent with an increase in the DFE of $\mathrm{V}_{\mathrm{Sn}^{2}}{ }^{2-}$ of $\sim 2$ times the VB shift (cf. eq 3 and the Supporting Information), i.e., to an increase in the $\mathrm{V}_{\mathrm{Sn}^{2-}}{ }^{2-} \mathrm{DFE}$ from $0.44 \mathrm{eV}$ in the pristine material to $0.44+0.10 / 0.14$ $\mathrm{eV}$. At the simulated $3 \%$ doping densities, a reduction in the hole density of $\sim 2$ orders of magnitude is accordingly predicted (from $\sim 10^{19}$ to $\sim 10^{17}$ ); thus, these doping elements may contribute to alleviating the self-p-doping in tin/iodine perovskites. The energetics of metal exchange, however, are only slightly favored in the case of $\mathrm{Cd}$ and $\mathrm{Ca}$ substitution. The small exchange reaction energies suggest that a significant excess of metal is required to induce its incorporation into the bulk, in agreement with the results of Adjokatse et al., who found a PL blue-shift, corresponding to the signature of incorporation of $\mathrm{Sr}$ into the bulk, only at a Sr precursor concentration of $>15 \%$.(66) In line with these observations, we also predict an increase in the band gap upon incorporation of Sr into bulk MASnl ${ }_{3}$.

We investigated by state-of-the-art DFT methods the defect chemistry of tin iodide perovskite by focusing on the trapping activity of native defects in this system and the origin of the self-p-doping. The emergence of self-p-doping in $\mathrm{MASnl}_{3}$, not observed in lead-based perovskites, is ascribed to the high stability of tin vacancies, which originated in the high valence band energy. Our defect analysis confirms many similarities in defect chemistry among $\mathrm{MASnl}_{3}, \mathrm{MAPb}_{0.5} \mathrm{Sn}_{0.5} \mathrm{I}_{3}$, and $\mathrm{MAPbl}_{3}$. The relative stability of defects in these systems is significantly modulated by the different VB energies. This feature remarkably affects the charge trap chemistries, dominated by iodine in $\mathrm{MAPbl}_{3}$ and tin in $\mathrm{MASnl}_{3}$. Interestingly, the intermediate value of the valence band in the mixed $\mathrm{MAPb}_{0.5} \mathrm{Sn}_{0.5} \mathrm{I}_{3}$ phase leads to the parallel suppression of deep hole and electron traps found in the full metal perovskites. Overall, these results show that strategies for increasing the efficiencies of tin perovskites should focus on increasing the inherent low ionization potential of the tin perovskite, responsible for the p-doping. In this regard, changes in the chemical compositions, such as alloying of tin with different metals, among which we identify cadmium as a good candidate, may represent a promising route toward the exploitation of these materials in lead-free solar cells.

\section{Computational Details}

Defects have been modeled in the $2 \times 2 \times 2$ supercells of tetragonal MASnl $\mathrm{MAPb}_{0.5} \mathrm{Sn}_{0.5} \mathrm{I}_{3}$, and $\mathrm{MAPbl}_{3}$ phases using the supercell approach. Equilibrium structures have been found by relaxing ion positions in the defect supercells by using the PBEO functional $(46,47)$ and including dispersion corrections through the DFT-D3 scheme.(48) In all cases, the cell parameters were fixed to the experimental values: for $\mathrm{MASnl}_{3}, a=b=8.758 \AA$ and $c=12.429 \AA$; for $\mathrm{MAPb}_{0.5} \mathrm{Sn}_{0.5} \mathrm{I}_{3}, a=b=8.855 \AA$ and $c=12.535 \AA$; for $\mathrm{MAPbl}_{3}, a=b=8.849 \AA$ and $c=12.642 \AA .(2)$

Hybrid functional PBEO calculations have been performed by using the freely available CP2K suite of codes, (67) keeping the fraction of Fock exchange $\alpha$ at its original value (0.25), and by including van der Waals interactions through the DFT-D3 scheme.(48) Calculations have been carried out with GoedeckerTeter-Hutter pseudopotentials, (68) double- $\zeta$ polarized basis sets for the wave functions, $(69)$ and a cutoff of 300 Ry for the expansion of the electron density in plane waves. We used the auxiliary density matrix method with the cFIT auxiliary basis set to accelerate the hybrid functional calculations.(70)

The energies of all calculated structures have been thus refined by using the HSEO6 functional(49) by including SOC and DFT-D3 dispersions(48) by using the Quantum Espresso package.(71) We used full relativistic norm-conserving pseudopotentials $\left(\mathrm{Pb}, 22\right.$ electrons, $5 s^{2}, 5 p^{6}, 5 d^{10}, 6 s^{2}, 6 p^{2} ; S n, 22$ electrons, $4 s^{2}$, $4 p^{6}, 4 d^{10}, 5 s^{2}, 5 p^{2} ; 1,7$ electrons, 5s $s^{2}, 5 p^{5} ; C, 4$ electrons, $2 s^{2}, 2 p^{2} ; N, 5$ electrons, $2 s^{2}, 2 p^{3} ; H, 1$ electron, $1 s^{1}$ ) with a cutoff on the wave functions of 40 Ry and 80 Ry on the Fock grid, sampling the Brillouin zone at the 
gamma point. An increased fraction of exact exchange has been included in the HSE06 functional $(\alpha=0.43)$. Such a value has been shown to provide accurate electronic properties of lead perovskites, in good agreement with experiments and state-of-the-art $\mathrm{G}_{0} \mathrm{~W}_{0}-\mathrm{SOC}$ calculations. $(10,33,52)$ Band gaps of 1.26, 1.45, and $1.65 \mathrm{eV}$ were obtained by this approach for $\mathrm{MASnl}_{3}, \mathrm{MAPb}_{0.5} \mathrm{Sn}_{0.5} \mathrm{l}_{3}$, and $\mathrm{MAPb}_{3}$, respectively.

Defect formation energies (DFE) and thermodynamic ionization levels (TIL) were calculated according to the following equations: $(33,45)$

$$
\begin{aligned}
& \operatorname{DFE}\left[\mathrm{X}^{q}\right]=E\left[\mathrm{X}^{q}\right]-E[\text { perf }]-\sum_{i} n_{i} \mu_{i}+q\left(\varepsilon_{\mathrm{VB}}+\varepsilon_{\mathrm{F}}\right)+E_{\text {corr }}^{q} \\
& \varepsilon\left(q / q^{\prime}\right)=\frac{E\left[\mathrm{X}^{q}\right]-E\left[\mathrm{X}^{q^{\prime}}\right]}{q^{\prime}-q}+\frac{E_{\mathrm{corr}}^{q}-E_{\mathrm{corr}}^{q^{\prime}}}{q^{\prime}-q}-\varepsilon_{\mathrm{VB}}
\end{aligned}
$$

where $E\left[X^{q}\right]$ is the energy of the supercell with defect $X$ in charge state $q, E($ perf) is the energy of the perfect (nondefective) supercell, $n$ and $\mu$ are the number and the chemical potentials of the species added to or subtracted from the nondefective system, respectively, and $\varepsilon_{\mathrm{VB}}$ is the valence band energy. The last two terms of eq 3 represent the energy due to the exchange of electrons with the Fermi level of the system $\left(\varepsilon_{\mathrm{F}}\right)$ and corrections due to periodic charges. Due to the high ionic dielectric constants of the simulated perovskites and the shallow nature of many defects, only the electrostatic potential correction has been included in the $E_{\text {corr }^{q}}$ term of the equations.

The chemical potentials of $\mathrm{MAPb}_{x} \mathrm{Sn}_{1-x} \mathrm{I}_{3}(x=0,0.5$, or 1$)$ constituents were set by considering the thermodynamic equilibrium between the perovskite and the relative metal precursors $\left(\mathrm{Snl}_{2}\right.$ and $\left.\mathrm{Pbl}_{2}\right)$

$$
\begin{aligned}
& \mu(\mathrm{MA})+x \mu(\mathrm{Pb})+(1-x) \mu(\mathrm{Sn})+3 \mu(I)=\mu\left(\mathrm{MAPb}_{x} \mathrm{Sn}_{1-x} \mathrm{I}_{3}\right) \\
& \mu(\mathrm{Pb})+2 \mu(\mathrm{I})=\mu\left(\mathrm{PbI}_{2}\right) \\
& \mu(\mathrm{Sn})+2 \mu(\mathrm{I})=\mu\left(\mathrm{SnI}_{2}\right)
\end{aligned}
$$

Three different chemical conditions of growth were simulated, i.e., iodine-rich, iodine-medium, and iodinepoor (points $\mathrm{A}-\mathrm{C}$ in Figure 1, respectively). I-Rich conditions were simulated by setting the chemical potentials of iodine to the value of solid $I_{2}$ for $\mathrm{MAPbl}_{3}\left[\mu(I)=1 / 2 \mu\left(I_{2}{ }^{\text {sol }}\right)\right]$, while due to the instability of $\mathrm{MASnl}_{3}$ under such conditions, the chemical potentials of the species of $\mathrm{MASnl}_{3}$ under I-rich conditions have been calculated by considering the equilibrium among the $\mathrm{MASnl}_{3}, \mathrm{Snl}_{4}$, and $\mathrm{Snl}_{2}$ phases (see Figure 1b).(72-74) I-Poor conditions were simulated by setting the chemical potentials of $\mathrm{Pb}$ and $\mathrm{Sn}$ to the values in the respective metal bulk phases $\left[\mu(\mathrm{Pb})=\mu\left(\mathrm{Pb}_{\text {bulk }}\right)\right.$, and $\left.\mu(\mathrm{Sn})=\mu\left(\mathrm{Sn}_{\text {bulk }}\right)\right]$. For the mixed $\mathrm{MAPb}_{0.5} \mathrm{Sn}_{0.5} \mathrm{l}_{3}$ phase, the same chemical potential ranges calculated for $\mathrm{Sn}$ and I in $\mathrm{MASnl}_{3}$ have been used. Band edge energies and TILs of $\mathrm{MASnl}_{3}, \mathrm{MAPb}_{0.5} \mathrm{Sn}_{0.5} \mathrm{I}_{3}$, and $\mathrm{MAPb}_{3}$ reported in Figure $5 \mathrm{~d}$ have been aligned to the inner $5 \mathrm{~s}$ and $4 \mathrm{~s}$ levels of $\mathrm{Pb}$ and $\mathrm{Sn}$, included in the norm-conserving pseudopotentials used in hybrid SOC calculations. 
The barrier to electron trapping on the $\mathrm{Sn}_{\mathrm{i}}{ }^{2+}$ defect has been calculated by performing a linear transit between the equilibrium configurations of the defect in the $2+$ and $1+$ charge states, by fixing the charge of the supercell to $1+$, i.e., simulating trapping of one electron on $\mathrm{Sn}_{i}{ }^{2+}$. In the linear transit, the $\mathrm{Sn}-\mathrm{Sn}$ distance between the tin interstitial and a tin of the lattice represents the reaction coordinate to the electron trapping process. The energy profile was calculated by using the Perdew-Burke-Ernzenhof (PBE) exchangecorrelation functional (75) in the $2 \times 2 \times 2$ supercell by constraining Sn-Sn distances to values between 3.08 and $4.03 \AA$ (representing the $\mathrm{Sn}-\mathrm{Sn}$ equilibrium distances in $\mathrm{Sn}_{i}{ }^{+}$and $\mathrm{Sn}_{\mathrm{i}}{ }^{2+}$, respectively, at the PBE level) and relaxing ion positions. PBE calculations were performed using ultrasoft pseudopotentials (Sn, 14 electrons, $4 d^{10}, 5 s^{2}, 5 p^{2} ; 1,7$ electrons, $5 s^{2}, 5 p^{5} ; C, 4$ electrons, $2 s^{2}, 2 p^{2} ; N, 5$ electrons, $2 s^{2}, 2 p^{3} ; H, 1$ electron, $1 \mathrm{~s}^{1}$ ) and the plane-wave basis set. A cutoff on the wave function of $25 \mathrm{Ry}$ and on the charge density of 200 Ryd was used, sampling the Brillouin zone at the gamma point. The calculated barrier at the PBE level was thus refined by performing single-point hybrid calculations using the HSE06-SOC functional ( $\alpha$ $=0.43$ ).

DFT calculations of doped $\mathrm{MASnl}_{3}$ have been performed in the $2 \times 2 \times 2$ supercell by simulating a doping percentage increasing from $3 \%$ to $25 \%$, by substituting from 1 to 8 tin ions in the pristine supercells ( 32 formula units). Equilibrium structures were computed using the PBE functional, (75) relaxing ion positions until forces on atoms were $<0.001 \mathrm{Ry} \AA^{-1}$ and fixing cell parameters to the experimental values. To quantitatively estimate defect properties, DFEs and electronic properties of singly substituted dopant supercells were refined by using the HSE06(49) hybrid functional $(\alpha=0.43)$, including SOC, and applying dispersion corrections a posteriori through the DFT-D3 scheme of Grimme.(48)

\section{Supporting Information}

The Supporting Information is available free of charge at https://pubs.acs.org/doi/10.1021/acs.jpclett.0c00725.

- Projected density of states (PDOS) computed at the HSEO6-SOC level of $\mathrm{MAPbl}_{3}$ and MASnl $\mathrm{M}_{3}$ structures of the reduced and oxidized forms of defects in $\mathrm{MASnl}_{3}$ and $\mathrm{MAPbl}_{3}$; calculated band edge energy shifts $\left(\triangle \mathrm{VB}\right.$ and $\triangle \mathrm{CB}$ ), band gaps $\left(E_{\mathrm{g}}\right)$, exchange energies, and variation of the DFEs of $\mathrm{V}_{\mathrm{Sn}^{2-}}{ }^{2-}$ of $\mathrm{MASnl}_{3}$ doped with $\mathrm{Cd}, \mathrm{Sr}, \mathrm{Zn}$, and $\mathrm{Ca}$ as substitutions for tin (PBE level); diagram reporting the relative variation of the tin vacancy $\mathrm{V}_{S n}{ }^{2-} \mathrm{DFE}(\mathrm{PBE})$ and $2 * \mathrm{VB}$ with respect to the pristine $\mathrm{MASn}_{3}$ for increasing dopant densities; converged structures and energies of the phases used throughout the work (Table S2); the converged $2 \times 2 \times 2$ supercell structures of $\mathrm{MAPbl}_{3}, \mathrm{MASnl}_{3}$, and $\mathrm{MAPb}_{0.5} \mathrm{Sn}_{0.5} \mathrm{l}_{3}$ (Table S3); and iodine interstitials $\mathrm{l}_{i}^{+}, \mathrm{l}_{\mathrm{i}}{ }^{0}$, and $\mathrm{l}_{i}^{-}$in $\mathrm{MAPbl}_{3}$ and tin interstitial $\mathrm{Sn}_{\mathrm{i}}{ }^{2+}$, $\mathrm{Sn}_{i}^{+}$, and $\mathrm{Sn}_{i}^{0}$ in $\mathrm{MASnl}_{3}(\underline{\mathrm{PDF}})$

\section{Terms \& Conditions}

Most electronic Supporting Information files are available without a subscription to ACS Web Editions. Such files may be downloaded by article for research use (if there is a public use license linked to the relevant article, that license may permit other uses). Permission may be obtained from ACS for other uses through requests via the RightsLink permission system: http://pubs.acs.org/page/copyright/permissions.html.

\section{Author Information}

\section{- Corresponding Authors}

- Daniele Meggiolaro - Computational Laboratory for Hybrid/Organic Photovoltaics (CLHYO), Istituto CNR di Scienze e Tecnologie Chimiche "Giulio Natta"(CNR-SCITEC), Via Elce di Sotto 8, $06123 \quad$ Perugia, Italy; http://orcid.org/0000-0001-9717133X; Email: daniele.meggiolaro@cnr.it 
- Filippo De Angelis - Computational Laboratory for Hybrid/Organic Photovoltaics (CLHYO), Istituto CNR di Scienze e Tecnologie Chimiche "Giulio Natta"(CNR-SCITEC), Via Elce di Sotto 8, 06123 Perugia, Italy; Department of Chemistry, Biology and Biotechnology, University of Perugia, Via Elce di Sotto 8, 06123 Perugia, Italy; Chemistry Department, College of Science, King Saud University, Riyadh 11451, Saudi Arabia; http://orcid.org/0000-0003-38331975; Email: filippo@thch.unipg.it

\section{- Authors}

- Damiano Ricciarelli - Computational Laboratory for Hybrid/Organic Photovoltaics (CLHYO), Istituto CNR di Scienze e Tecnologie Chimiche "Giulio Natta"(CNR-SCITEC), Via Elce di Sotto 8, 06123 Perugia, Italy; Department of Chemistry, Biology and Biotechnology, University of Perugia, Via Elce di Sotto 8, 06123 Perugia, Italy

- Ahmed A. Alasmari-The First Industrial Institute, TVTC, Riyadh 11451, Saudi Arabia; Physics and Astronomy Department, College of Science, King Saud University, Riyadh 11451, Saudi Arabia

- Fatmah A. S. Alasmary - Chemistry Department, College of Science, King Saud University, Riyadh 11451, Saudi Arabia

\section{- Notes}

The authors declare no competing financial interest.

\section{Acknowledgments}

This work was supported by the Distinguished Scientist Fellowship Program (DSFP) of King Saud University. The authors acknowledge support from the Ministero Istruzione dell'Università e della Ricerca (MIUR) and the University of Perugia through the program "Dipartimenti di Eccellenza 2018-2022" (grant AMIS) and from the European Union's Horizon 2020 research and innovation program under Grant Agreement 764047 of the Espresso project.

\section{References}

This article references 75 other publications.

1. Green, M. A.; Ho-Baillie, A.; Snaith, H. J. The Emergence of Perovskite Solar Cells. Nat. Photonics 2014, 8, 506- 514, DOI: 10.1038/nphoton.2014.134

2. Stoumpos, C. C.; Malliakas, C. D.; Kanatzidis, M. G. Semiconducting Tin and Lead lodide Perovskites with Organic Cations: Phase Transitions, High Mobilities, and near-Infrared Photoluminescent Properties. Inorg. Chem. 2013, 52, 9019-9038, DOI: 10.1021/ic401215x

3. Stranks, S. D.; Eperon, G. E.; Grancini, G.; Menelaou, C.; Alcocer, M. J. P.; Leijtens, T.; Herz, L. M.; Petrozza, A.; Snaith, H. J. Electron-Hole Diffusion Lengths Exceeding 1 Micrometer in an Organometal Trihalide Perovskite Absorber. Science 2013, 342, 341-344, DOI: 10.1126/science.1243982

4. Miyata, A.; Mitioglu, A.; Plochocka, P.; Portugall, O.; Wang, J. T.-W.; Stranks, S. D.; Snaith, H. J.; Nicholas, R. J. Direct Measurement of the Exciton Binding Energy and Effective Masses for Charge Carriers in Organic-Inorganic Tri-Halide Perovskites. Nat. Phys. 2015, 11, 582-587, DOI: $10.1038 /$ nphys3357 
5. Lin, Q.; Armin, A.; Nagiri, R. C. R.; Burn, P. L.; Meredith, P. Electro-Optics of Perovskite Solar Cells. Nat. Photonics 2015, 9, 106, DOI: 10.1038/nphoton.2014.284

6. Meggiolaro, D.; Motti, S. G.; Mosconi, E.; Barker, A. J.; Ball, J.; Andrea Riccardo Perini, C.; Deschler, F.; Petrozza, A.; De Angelis, F. lodine Chemistry Determines the Defect Tolerance of Lead-Halide Perovskites. Energy Environ. Sci. 2018, 11, 702- 713, DOI: 10.1039/C8EE00124C

7. NREL. Best Research-Cell Efficiencies, 06-08-2019. https://www.nrel.gov/pv/assets/pdfs/bestresearch-cell-efficiencies.20190802.pdf, 2019.

8. Yin, W.-J.; Shi, T.; Yan, Y. Unusual Defect Physics in Ch3nh3pbi3 Perovskite Solar Cell Absorber. Appl. Phys. Lett. 2014, 104, 063903 DOI: 10.1063/1.4864778

9. Buin, A.; Comin, R.; Xu, J.; Ip, A. H.; Sargent, E. H. Halide-Dependent Electronic Structure of Organolead Perovskite Materials. Chem. Mater. 2015, 27, 4405-4412, DOI: 10.1021/acs.chemmater.5b01909

10. Du, M.-H. Density Functional Calculations of Native Defects in Ch3nh3pbi3: Effects of Spin-Orbit Coupling and Self-Interaction Error.J. Phys. Chem. Lett. 2015, 6, 1461-1466, DOI: 10.1021/acs.jpclett.5b00199

11. Wiktor, J.; Ambrosio, F.; Pasquarello, A. Mechanism Suppressing Charge Recombination at lodine Defects in Ch3nh3pbi3 by Polaron Formation. J. Mater. Chem. A 2018, 6, 16863-16867, DOI: 10.1039/C8TA06466K

12. Babayigit, A.; Ethirajan, A.; Muller, M.; Conings, B. Toxicity of Organometal Halide Perovskite Solar Cells. Nat. Mater. 2016, 15, 247, DOI: 10.1038/nmat4572

13. Li, J.; Cao, H.-L.; Jiao, W.-B.; Wang, Q.; Wei, M.; Cantone, I.; Lü, J.; Abate, A. Biological Impact of Lead from Halide Perovskites Reveals the Risk of Introducing a Safe Threshold. Nat. Commun. 2020, 11, 310, DOI: 10.1038/s41467-019-13910-y

14. Giustino, F.; Snaith, H. J. Toward Lead-Free Perovskite Solar Cells. ACS Energy Lett. 2016, 1, 12331240, DOI: 10.1021/acsenergylett.6b00499

15. Noel, N. K.; Stranks, S. D.; Abate, A.; Wehrenfennig, C.; Guarnera, S.; Haghighirad, A.-A.; Sadhanala, A.; Eperon, G. E.; Pathak, S. K.; Johnston, M. B.Lead-Free Organic-Inorganic Tin Halide Perovskites for Photovoltaic Applications. Energy Environ. Sci. 2014, 7, 3061- 3068, DOI: 10.1039/C4EE01076K

16. Konstantakou, M.; Stergiopoulos, T. A Critical Review on Tin Halide Perovskite Solar Cells. J. Mater. Chem. A 2017, 5, 11518-11549, DOI: 10.1039/C7TA00929A

17. Stoumpos, C. C.; Frazer, L.; Clark, D. J.; Kim, Y. S.; Rhim, S. H.; Freeman, A. J.; Ketterson, J. B.; Jang, J. I.; Kanatzidis, M. G. Hybrid Germanium lodide Perovskite Semiconductors: Active Lone Pairs, Structural Distortions, Direct and Indirect Energy Gaps, and Strong Nonlinear Optical Properties. J. Am. Chem. Soc. 2015, 137, 6804-6819, DOI: 10.1021/jacs.5b01025

18. McClure, E. T.; Ball, M. R.; Windl, W.; Woodward, P. M. Cs2agbix6 (X = Br, Cl): New Visible Light Absorbing, Lead-Free Halide Perovskite Semiconductors. Chem. Mater. 2016, 28, 1348-1354, DOI: 10.1021/acs.chemmater.5b04231

19. Filip, M. R.; Hillman, S.; Haghighirad, A. A.; Snaith, H. J.; Giustino, F. Band Gaps of the Lead-Free Halide Double Perovskites Cs2biagcl6 and Cs2biagbr6 from Theory and Experiment. J. Phys. Chem. Lett. 2016, 7, 2579-2585, DOI: 10.1021/acs.jpclett.6b01041 
20. Slavney, A. H.; Hu, T.; Lindenberg, A. M.; Karunadasa, H. I. A Bismuth-Halide Double Perovskite with Long Carrier Recombination Lifetime for Photovoltaic Applications.J. Am. Chem. Soc. 2016, 138, 2138-2141, DOI: 10.1021/jacs.5b13294

21. Kumar, M. H.; Dharani, S.; Leong, W. L.; Boix, P. P.; Prabhakar, R. R.; Baikie, T.; Shi, C.; Ding, H.; Ramesh, R.; Asta, M.Lead-Free Halide Perovskite Solar Cells with High Photocurrents Realized through Vacancy Modulation. Adv. Mater. 2014, 26, 7122-7127, DOI: 10.1002/adma.201401991

22. Tong, J.; Song, Z.; Kim, D. H.; Chen, X.; Chen, C.; Palmstrom, A. F.; Ndione, P. F.; Reese, M. O.; Dunfield, S. P.; Reid, O. G.Carrier Lifetimes of $>1 \mathrm{Ms}$ in Sn-Pb Perovskites Enable Efficient AllPerovskite Tandem Solar Cells. Science 2019, 364, 475-479, DOI: 10.1126/science.aav7911

23. Xiao, Z.; Song, Z.; Yan, Y. From Lead Halide Perovskites to Lead-Free Metal Halide Perovskites and Perovskite Derivatives. Adv. Mater. 2019, 31, 1803792, DOI: 10.1002/adma.201803792

24. Zhao, D.; Yu, Y.; Wang, C.; Liao, W.; Shrestha, N.; Grice, C. R.; Cimaroli, A. J.; Guan, L.; Ellingson, R. J.; Zhu, K.Low-Bandgap Mixed Tin-Lead Iodide Perovskite Absorbers with Long Carrier Lifetimes for All-Perovskite Tandem Solar Cells. Nat. Energy 2017, 2, 17018, DOI: 10.1038/nenergy.2017.18

25. Milot, R. L.; Klug, M. T.; Davies, C. L.; Wang, Z.; Kraus, H.; Snaith, H. J.; Johnston, M. B.; Herz, L. M. The Effects of Doping Density and Temperature on the Optoelectronic Properties of Formamidinium Tin Triiodide Thin Films. Adv. Mater. 2018, 30, 1804506, DOI: 10.1002/adma.201804506

26. Takahashi, Y.; Obara, R.; Lin, Z.-Z.; Takahashi, Y.; Naito, T.; Inabe, T.; Ishibashi, S.; Terakura, K. Charge-Transport in Tin-lodide Perovskite Ch3nh3sni3: Origin of High Conductivity. Dalton Trans. 2011, 40, 5563- 5568, DOI: 10.1039/c0dt01601b

27. Chung, I.; Lee, B.; He, J.; Chang, R. P. H.; Kanatzidis, M. G. All-Solid-State Dye-Sensitized Solar Cells with High Efficiency. Nature 2012, 485, 486- 489, DOI: 10.1038/nature11067

28. Dalpian, G. M.; Liu, Q.; Stoumpos, C. C.; Douvalis, A. P.; Balasubramanian, M.; Kanatzidis, M. G.; Zunger, A. Changes in Charge Density Vs Changes in Formal Oxidation States: The Case of Sn Halide Perovskites and Their Ordered Vacancy Analogues. Phys. Rev. Materials 2017, 1, 025401 DOI: 10.1103/PhysRevMaterials.1.025401

29. Bowman, A.; Klug, M.; Doherty, T.; Farrar, M.; Senanayak, S. P.; Wenger, B.; Divitini, G.; Booker, E.; Andaji-Garmaroudi, Z.; Macpherson, S.Microsecond Carrier Lifetimes, Controlled P-Doping and Enhanced Air Stability in Low-Bandgap Metal Halide Perovskites. ACS Energy Lett. 2019, 4, 2301, DOI: 10.1021/acsenergylett.9b01446

30. Shao, S.; Liu, J.; Portale, G.; Fang, H.-H.; Blake, G. R.; ten Brink, G. H.; Koster, L. J. A.; Loi, M. A. Highly Reproducible Sn-Based Hybrid Perovskite Solar Cells with 9\% Efficiency. Adv. En. Mater. 2018, 8, 1702019, DOI: 10.1002/aenm.201702019

31. Leijtens, T.; Prasanna, R.; Gold-Parker, A.; Toney, M. F.; McGehee, M. D. Mechanism of Tin Oxidation and Stabilization by Lead Substitution in Tin Halide Perovskites. ACS Energy Lett. 2017, 2, 2159-2165, DOI: 10.1021/acsenergylett.7b00636

32. Ahuja, R.; Blomqvist, A.; Larsson, P.; Pyykkö, P.; Zaleski-Ejgierd, P. Relativity and the Lead-Acid Battery. Phys. Rev. Lett. 2011, 106, 018301 DOI: 10.1103/PhysRevLett.106.018301

33. Meggiolaro, D.; De Angelis, F. First-Principles Modeling of Defects in Lead Halide Perovskites: Best Practices and Open Issues. ACS Energy Lett. 2018, 3, 2206-2222, DOI: 10.1021/acsenergylett.8b01212 
34. Lee, S. J.; Shin, S. S.; Kim, Y. C.; Kim, D.; Ahn, T. K.; Noh, J. H.; Seo, J.; Seok, S. I. Fabrication of Efficient Formamidinium Tin lodide Perovskite Solar Cells through Snf2-Pyrazine Complex. J. Am. Chem. Soc. 2016, 138, 3974-3977, DOI: 10.1021/jacs.6b00142

35. Hoshi, H.; Shigeeda, N.; Dai, T. Improved Oxidation Stability of Tin lodide Cubic Perovskite Treated by 5-Ammonium Valeric Acid lodide.Mater. Lett. 2016, 183, 391-393, DOI: 10.1016/j.matlet.2016.07.048

36. Tai, Q.; Guo, X.; Tang, G.; You, P.; Ng, T.-W.; Shen, D.; Cao, J.; Liu, C.-K.; Wang, N.; Zhu, Y.Antioxidant Grain Passivation for Air-Stable Tin-Based Perovskite Solar Cells. Angew. Chem., Int. Ed. 2019, 58, 806-810, DOI: 10.1002/anie.201811539

37. Song, T.-B.; Yokoyama, T.; Stoumpos, C. C.; Logsdon, J.; Cao, D. H.; Wasielewski, M. R.; Aramaki, S.; Kanatzidis, M. G. Importance of Reducing Vapor Atmosphere in the Fabrication of Tin-Based Perovskite Solar Cells. J. Am. Chem. Soc. 2017, 139, 836-842, DOI: 10.1021/jacs.6b10734

38. Li, W.; Li, J.; Li, J.; Fan, J.; Mai, Y.; Wang, L. Addictive-Assisted Construction of All-Inorganic Cssnibr2Mesoscopic Perovskite Solar Cells with Superior Thermal Stability up to $473 \mathrm{~K}$. J. Mater. Chem. A 2016, 4, 17104-17110, DOI: 10.1039/C6TA08332C

39. Kayesh, M. E.; Matsuishi, K.; Kaneko, R.; Kazaoui, S.; Lee, J.-J.; Noda, T.; Islam, A. Coadditive Engineering with 5-Ammonium Valeric Acid lodide for Efficient and Stable Sn Perovskite Solar Cells. ACS Energy Lett. 2019, 4, 278-284, DOI: 10.1021/acsenergylett.8b02216

40. Jokar, E.; Chien, C.-H.; Fathi, A.; Rameez, M.; Chang, Y.-H.; Diau, E. W.-G. Slow Surface Passivation and Crystal Relaxation with Additives to Improve Device Performance and Durability for Tin-Based Perovskite Solar Cells. Energy Environ. Sci. 2018, 11, 2353-2362, DOI: 10.1039/C8EE00956B

41. Liao, Y.; Liu, H.; Zhou, W.; Yang, D.; Shang, Y.; Shi, Z.; Li, B.; Jiang, X.; Zhang, L.; Quan, L. N.Highly Oriented Low-Dimensional Tin Halide Perovskites with Enhanced Stability and Photovoltaic Performance. J. Am. Chem. Soc. 2017, 139, 6693-6699, DOI: 10.1021/jacs.7b01815

Xu, P.; Chen, S.; Xiang, H.-J.; Gong, X.-G.; Wei, S.-H. Influence of Defects and Synthesis Conditions on the Photovoltaic Performance of Perovskite Semiconductor Cssni3.Chem. Mater. 2014, 26, 6068-6072, DOI: 10.1021/cm503122j

42. Shi, T.; Zhang, H.-S.; Meng, W.; Teng, Q.; Liu, M.; Yang, X.; Yan, Y.; Yip, H.-L.; Zhao, Y.-J. Effects of Organic Cations on the Defect Physics of Tin Halide Perovskites. J. Mater. Chem. A 2017, 5, 1512415129, DOI: 10.1039/C7TA02662E

43. Mosconi, E.; Umari, P.; De Angelis, F. Electronic and Optical Properties of Mixed Sn-Pb Organohalide Perovskites: A First Principles Investigation. J. Mater. Chem. A 2015, 3, 92089215, DOI: 10.1039/C4TA06230B

44. Van de Walle, C. G.; Neugebauer, J. First-Principles Calculations for Defects and Impurities: Applications to lii-Nitrides. J. Appl. Phys. 2004, 95, 3851-3879, DOI: 10.1063/1.1682673

45. Perdew, J. P.; Ernzerhof, M.; Burke, K. Rationale for Mixing Exact Exchange with Density Functional Approximations. J. Chem. Phys. 1996, 105, 9982-9985, DOI: 10.1063/1.472933

46. Adamo, C.; Barone, V. Toward Reliable Density Functional Methods without Adjustable Parameters: The Pbe0Model. J. Chem. Phys. 1999, 110, 6158-6170, DOI: 10.1063/1.478522 
47. Grimme, S.; Antony, J.; Ehrlich, S.; Krieg, H. A Consistent and Accurate Ab Initio Parametrization of Density Functional Dispersion Correction (Dft-D) for the 94 Elements H-Pu.J. Chem. Phys. 2010, 132, 154104, DOI: 10.1063/1.3382344

48. Heyd, J.; Scuseria, G. E.; Ernzerhof, M. Hybrid Functionals Based on a Screened Coulomb Potential. J. Chem. Phys. 2003, 118, 8207-8215, DOI: 10.1063/1.1564060

49. Meggiolaro, D.; Mosconi, E.; De Angelis, F. Formation of Surface Defects Dominates Ion Migration in Lead-Halide Perovskites. ACS Energy Lett. 2019, 4, 779- 785, DOI: 10.1021/acsenergylett.9b00247

50. Chen, B.; Li, T.; Dong, Q.; Mosconi, E.; Song, J.; Chen, Z.; Deng, Y.; Liu, Y.; Ducharme, S.; Gruverman, A.Large Electrostrictive Response in Lead Halide Perovskites. Nat. Mater. 2018, 17, 10201026, DOI: 10.1038/s41563-018-0170-x

51. Umari, P.; Mosconi, E.; De Angelis, F. Relativistic Gw Calculations on $\mathrm{Ch}_{3} \mathrm{nh}_{3} \mathrm{pbi}_{3}$ and $\mathrm{Ch}_{3} \mathrm{nh}_{3} \mathrm{Sni}_{3}$ Perovskites for Solar Cell Applications. Sci. Rep. 2015, 4, 4467, DOI: 10.1038/srep04467

52. Tao, S.; Schmidt, I.; Brocks, G.; Jiang, J.; Tranca, I.; Meerholz, K.; Olthof, S. Absolute Energy Level Positions in Tin- and Lead-Based Halide Perovskites. Nat. Commun. 2019, 10, 2560, DOI: $10.1038 / \mathrm{s} 41467-019-10468-7$

53. Meggiolaro, D.; Mosconi, E.; Proppe, A. H.; Quintero-Bermudez, R.; Kelley, S. O.; Sargent, E. H.; De Angelis, F. Energy Level Tuning at the Mapbi3 Perovskite/Contact Interface Using Chemical Treatment. ACS Energy Lett. 2019, 4, 2181-2184, DOI: 10.1021/acsenergylett.9b01584

54. Agiorgousis, M. L.; Sun, Y.-Y.; Zeng, H.; Zhang, S. Strong Covalency-Induced Recombination Centers in Perovskite Solar Cell Material Ch3nh3pbi3. J. Am. Chem. Soc. 2014, 136, 14570-14575, DOI: 10.1021/ja5079305

55. Liao, W.; Zhao, D.; Yu, Y.; Grice, C. R.; Wang, C.; Cimaroli, A. J.; Schulz, P.; Meng, W.; Zhu, K.; Xiong, R.-G.Lead-Free Inverted Planar Formamidinium Tin Triiodide Perovskite Solar Cells Achieving Power Conversion Efficiencies up to 6.22\%. Adv. Mater. 2016, 28, 9333-9340, DOI: 10.1002/adma.201602992

56. Sabba, D.; Mulmudi, H. K.; Prabhakar, R. R.; Krishnamoorthy, T.; Baikie, T.; Boix, P. P.; Mhaisalkar, S.; Mathews, N. Impact of Anionic $\mathrm{Br}$ - Substitution on Open Circuit Voltage in Lead Free Perovskite (Cssni3-Xbrx) Solar Cells. J. Phys. Chem. C 2015, 119, 1763- 1767, DOI: 10.1021/jp5126624

57. Xing, G.; Kumar, M. H.; Chong, W. K.; Liu, X.; Cai, Y.; Ding, H.; Asta, M.; Grätzel, M.; Mhaisalkar, S.; Mathews, N.Solution-Processed Tin-Based Perovskite for near-Infrared Lasing. Adv. Mater. 2016, 28, 8191-8196, DOI: 10.1002/adma.201601418

58. Koh, T. M.; Krishnamoorthy, T.; Yantara, N.; Shi, C.; Leong, W. L.; Boix, P. P.; Grimsdale, A. C.; Mhaisalkar, S. G.; Mathews, N. Formamidinium Tin-Based Perovskite with Low Eg for Photovoltaic Applications. J. Mater. Chem. A 2015, 3, 14996-15000, DOI: 10.1039/C5TA00190K

59. Bowman, A. R.; Klug, M. T.; Doherty, T. A. S.; Farrar, M. D.; Senanayak, S. P.; Wenger, B.; Divitini, G.; Booker, E. P.; Andaji-Garmaroudi, Z.; Macpherson, S.Microsecond Carrier Lifetimes, Controlled P-Doping, and Enhanced Air Stability in Low-Bandgap Metal Halide Perovskites. ACS Energy Lett. 2019, 4, 2301-2307, DOI: 10.1021/acsenergylett.9b01446

60. Pérez-del-Rey, D.; Forgács, D.; Hutter, E. M.; Savenije, T. J.; Nordlund, D.; Schulz, P.; Berry, J. J.; Sessolo, M.; Bolink, H. J. Strontium Insertion in Methylammonium Lead lodide: Long Charge 
Carrier Lifetime and High Fill-Factor Solar Cells. Adv. Mater. 2016, 28, 9839-9845, DOI: 10.1002/adma.201603016

61. Lau, C. F. J.; Zhang, M.; Deng, X.; Zheng, J.; Bing, J.; Ma, Q.; Kim, J.; Hu, L.; Green, M. A.; Huang, S.Strontium-Doped Low-Temperature-Processed Cspbi2br Perovskite Solar Cells. ACS Energy Lett. 2017, 2, 2319-2325, DOI: 10.1021/acsenergylett.7b00751

62. Zhang, H.; Wang, H.; Williams, S. T.; Xiong, D.; Zhang, W.; Chueh, C.-C.; Chen, W.; Jen, A. K.-Y. Srcl2 Derived Perovskite Facilitating a High Efficiency of $16 \%$ in Hole-Conductor-Free Fully Printable Mesoscopic Perovskite Solar Cells. Adv. Mater. 2017, 29, 1606608, DOI: 10.1002/adma.201606608

63. Hao, F.; Stoumpos, C. C.; Cao, D. H.; Chang, R. P. H.; Kanatzidis, M. G. Lead-Free Solid-State OrganicInorganic Halide Perovskite Solar Cells. Nat. Photonics 2014, 8, 489-494, DOI: 10.1038/nphoton.2014.82

64. Lee, S. J.; Shin, S. S.; Im, J.; Ahn, T. K.; Noh, J. H.; Jeon, N. J.; Seok, S. I.; Seo, J. Reducing Carrier Density in Formamidinium Tin Perovskites and Its Beneficial Effects on Stability and Efficiency of Perovskite Solar Cells. ACS Energy Lett. 2018, 3, 46- 53, DOI: 10.1021/acsenergylett.7b00976

65. Adjokatse, S.; Kahmann, S.; Duim, H.; Loi, M. A. Effects of Strontium Doping on the Morphological, Structural, and Photophysical Properties of Fasni3 Perovskite Thin Films. APL Mater. 2019, 7, 031116 DOI: 10.1063/1.5087110

66. VandeVondele, J.; Krack, M.; Mohamed, F.; Parrinello, M.; Chassaing, T.; Hutter, J. Quickstep: Fast and Accurate Density Functional Calculations Using a Mixed Gaussian and Plane Waves Approach. Comput. Phys. Commun. 2005, 167, 103-128, DOI: 10.1016/j.cpc.2004.12.014

67. Goedecker, S.; Teter, M.; Hutter, J. Separable Dual-Space Gaussian Pseudopotentials. Phys. Rev. B: Condens. Matter Mater. Phys. 1996, 54, 1703-1710, DOI: 10.1103/PhysRevB.54.1703

68. VandeVondele, J.; Hutter, J. Gaussian Basis Sets for Accurate Calculations on Molecular Systems in Gas and Condensed Phases. J. Chem. Phys. 2007, 127, 114105, DOI: 10.1063/1.2770708

69. Guidon, M.; Hutter, J.; VandeVondele, J.Auxiliary Density Matrix Methods for Hartree-Fock Exchange Calculations. J. Chem. Theory Comput. 2010, 6, 2348-2364, DOI: 10.1021/ct1002225

70. Giannozzi, P.; Baroni, S.; Bonini, N.; Calandra, M.; Car, R.; Cavazzoni, C.; Ceresoli, D.; Chiarotti, G. L.; Coccioni, M.; Dabo, I.Quantum Espresso: A Modular and Open-Source Software Project for Quantum Simulations of Materials.J. Phys.: Condens. Matter 2009, 21, 395502, DOI: 10.1088/0953-8984/21/39/395502

71. Moser, W.; Trevena, I. C. The Crystal Structure of Tin(li) lodide. J. Chem. Soc. D 1969, 25- 26, DOI: $10.1039 / \mathrm{c} 29690000025$

72. Groth, P.; Kaiser, E.Zeitschrift Für Kristallographie, Kristallgeometrie, Kristallphysik, Kristallchemie; Akademische Verlagsgesellschaft, 1896; Vol. 26.

73. Funabiki, F.; Toda, Y.; Hosono, H. Optical and Electrical Properties of Perovskite Variant (Ch3nh3)2sni6. J. Phys. Chem. C 2018, 122, 10749-10754, DOI: 10.1021/acs.jpcc.8b01820

74. Perdew, J. P.; Burke, K.; Ernzerhof, M. Generalized Gradient Approximation Made Simple. Phys. Rev. Lett. 1996, 77, 3865-3868, DOI: 10.1103/PhysRevLett.77.3865 